\title{
Chemical screen identifies a geroprotective role of quercetin in premature aging
}

\author{
Lingling Geng ${ }^{1,2}$, Zunpeng Liu ${ }^{3,5}$, Weiqi Zhang ${ }^{1,2,5}$, Wei $\mathrm{Li}^{1,3}$, Zeming $\mathrm{Wu}^{3,5}$, Wei Wang ${ }^{2,5}$, Ruotong Ren ${ }^{2,5}$, \\ Yao Su ${ }^{1}$, Peichang Wang ${ }^{1}$, Liang Sun ${ }^{8}$, Zhenyu Ju', Piu Chan ${ }^{1,5}$, Moshi Song ${ }^{4,5,6 凶}$, Jing Qu ${ }^{3,5,6 \bowtie}$, \\ Guang-Hui Liu $^{1,2,5,6,7 凶}$ (D) \\ ${ }^{1}$ Advanced Innovation Center for Human Brain Protection, National Clinical Research Center for Geriatric Disorders, Xuanwu \\ Hospital of Capital Medical University, Beijing 100053, China \\ ${ }^{2}$ National Laboratory of Biomacromolecules, CAS Center for Excellence in Biomacromolecules, Institute of Biophysics, \\ Chinese Academy of Sciences, Beijing 100101, China \\ ${ }^{3}$ State Key Laboratory of Stem Cell and Reproductive Biology, Institute of Zoology, Chinese Academy of Sciences, Beijing \\ 100101, China \\ ${ }^{4}$ State Key Laboratory of Membrane Biology, Institute of Zoology, Chinese Academy of Sciences, Beijing 100101, China \\ ${ }^{5}$ University of Chinese Academy of Sciences, Beijing 100049, China \\ ${ }^{6}$ Institute of Stem cell and Regeneration, Chinese Academy of Sciences, Beijing 100101, China \\ ${ }^{7}$ Key Laboratory of Regenerative Medicine of Ministry of Education, Institute of Aging and Regenerative Medicine, Jinan \\ University, Guangzhou 510632, China \\ 8 The MOH Key Laboratory of Geriatrics, Beijing Hospital, National Center of Gerontology, Beijing 100730, China \\ $\square$ Correspondence: weiqizhang@aliyun.com (W. Zhang), songmoshi@ioz.ac.cn (M. Song), qujing@ioz.ac.cn (J. Qu), \\ ghliu@ibp.ac.cn (G.-H. Liu)
}

Received March 12, 2018 Accepted June 25, 2018

\begin{abstract}
Aging increases the risk of various diseases. The main goal of aging research is to find therapies that attenuate aging and alleviate aging-related diseases. In this study, we screened a natural product library for geroprotective compounds using Werner syndrome (WS) human mesenchymal stem cells (hMSCs), a premature aging model that we recently established. Ten candidate compounds were identified and quercetin was investigated in detail due to its leading effects. Mechanistic studies revealed that quercetin alleviated senescence via the enhancement of cell proliferation and restoration of heterochromatin architecture in WS hMSCs. RNA-sequencing analysis revealed the transcriptional commonalities and differences in the geroprotective effects by quercetin and Vitamin C. Besides WS hMSCs, quercetin also
\end{abstract}

Lingling Geng, Zunpeng Liu and Weiqi Zhang have contributed equally to this work.

Electronic supplementary material The online version of this article (https://doi.org/10.1007/s13238-018-0567-y) contains supplementary material, which is available to authorized users. attenuated cellular senescence in Hutchinson-Gilford progeria syndrome (HGPS) and physiological-aging hMSCs. Taken together, our study identifies quercetin as a geroprotective agent against accelerated and natural aging in hMSCs, providing a potential therapeutic intervention for treating age-associated disorders.

KEYWORDS Quercetin, Stem cell, Aging, Werner syndrome, Hutchinson-Gilford progeria syndrome

\section{INTRODUCTION}

Aging is associated with progressive functional decline over time at cellular, tissue and organismal levels, leading to increased vulnerability to diseases that include cancer, neurodegenerative disorders, as well as cardiovascular and metabolic diseases (Benayoun et al., 2015; Campisi, 2013; Kudlow et al., 2007; López-Otín et al., 2013). Possible mechanisms for aging include free radicals, telomere shortening (Harley, 1991), programmed senescence (Smith and Pereirasmith, 1996), genomic instability (Lombard et al., 2005), and loss of heterochromatin architecture (Benayoun et al., 2015; Kubben et al., 2016; Ren et al., 2017a; Ren et al., 2017b; Villeponteau, 1997; Zhang et al., 2015). On the 
basis of numerous studies having unraveled possible mechanisms of aging (Uccelli et al., 2008), continuous efforts are put into the identification of many novel targets with minimal side effects (López-Otín et al., 2013). In particular, drug screening for geroprotective chemicals has attracted curiosity and excited imagination throughout the history of humankind.

Progeroid syndromes are a group of rare genetic disorders characterized by clinical features of premature aging. Werner syndrome (WS) and Hutchinson-Gilford progeria syndrome (HGPS) are two best characterized types of progeria. WS is known as adult progeria, mainly characterized by premature aging pathologies associated with the degeneration of mesodermal tissues, resulting in symptoms such as osteoporosis and atherosclerosis (Opresko et al., 2003; Ozgenc and Loeb, 2006). WS is caused by mutated WRN gene, resulting in the loss of WRN protein expression. WRN participates in a continuum of cellular processes, spanning from DNA replication, transcription, repair, recombination as well as heterochromatin maintenance at telomeric and centromeric regions, thus pointing WS pathogenesis to genomic and epigenomic instability (Kudlow et al., 2007; Lebel, 2001; Li et al., 2016b; Wu et al., 2018; Zhang et al., 2015). The other well-studied progeria, HGPS is a devastating incurable disease with an average age of death at 14.6 years (Hennekam Raoul, 2006; Kreienkamp et al., 2016; Ullrich and Gordon, 2015). It is caused by the accumulation of progerin, a truncated protein encoded by a GGC>GGT (G608G) single-base mutation in LMNA gene instead of the functional nuclear lamina protein Lamin $A$ by wild-type LMNA gene. HGPS patient-derived cells usually exhibit nuclear morphological abnormalities, altered signaling pathways, genomic and epigenetic instability and premature senescence (Burtner and Kennedy, 2010; Kubben et al., 2016; Kudlow et al., 2007; López-Otín et al., 2013; Liu et al., 2011a; Liu et al., 2011b; Polosak et al., 2011; Ren et al., 2017b; Wu et al., 2018; Yang et al., 2014).

WS and HGPS are both relevant disease models for understanding the mechanisms of aging and for finding effective treatments for aging-associated disorders. We previously reported that Vitamin C (VC) rejuvenates WS hMSCs by inducing a global change in the transcriptome of aging-related genes (Li et al., 2016b). In addition, Vitamin D is reported to improve HGPS cellular phenotypes by reducing progerin production and stabilizing key factors for maintaining genome integrity (Kreienkamp et al., 2016). We recently established a human stem-cell-based platform with isogenic WS and HGPS hMSCs with the identical genetic background for the comparative mechanistic studies of aging as well as the identification of new drug discovery strategies for progeria syndromes and physiological aging (Kubben et al., 2016; Li et al., 2016b; Liu et al., 2011a; Liu et al., 2011b; Wu et al., 2018; Zhang et al., 2015).

In this study, we screened a natural product library for geroprotective drugs using WS hMSCs and identified ten candidate compounds including quercetin. Mechanistically,
Figure 1. Screening of a natural product library in WS hMSCs. (A) Schematics of the screening strategy of a natural product library for geroprotective drugs. (B) Results from MTS assay highlighting ten candidate compounds $(n=5) .{ }^{* *} P<0.001$ vs. vehicle. (C) Relative cell proliferative abilities upon the treatment of the ten candidate compounds at indicated concentrations $(n=3)$.

quercetin alleviated hMSC senescence by promoting the self-renewal and differentiation abilities as well as restoring the heterochromatin architecture of WS hMSCs. RNA-sequencing analysis revealed that quercetin rejuvenated WS hMSCs via the regulation of multiple cellular processes related to cell cycle, chromosome condensation and anti-oxidation, which were of mechanistic commonalities for VC-mediated geroprotective effects in WS hMSCs. In addition, quercetin rejuvenated HGPS as well as physiological-aging hMSCs. Taken together, these data indicate quercetin as a geroprotective agent against premature and physiological human aging.

\section{RESULTS} Natural product screening using Werner syndrome
hMSCs

We have recently established a platform of Werner syndrome (WS) and Hutchinson-Gilford progeria syndrome (HGPS) hMSCs for studying premature and physiological aging (Fang et al., 2018; Kubben et al., 2016; Li et al., 2016b; Wu et al., 2018; Yang et al., 2017; Zhang et al., 2015). Here, we took advantage of this platform and screened for natural product compounds capable of alleviating premature senescence (Fig. 1A). A total of 133 natural products (Table S1) were evaluated using WS hMSCs through a short-term treatment of each compound at $1 \mu \mathrm{mol} / \mathrm{L}$ for seven days. The effect on cell proliferation by each compound was evaluated by 3-(4,5-dimethylthiazol-2-yl)-5(3-carboxymethoxyphenyl)-2-(4-sulfophenyl)-2H-tetrazolium, inner salt (MTS) assay and ten compounds including quercetin dihydrate (referred to as quercetin, Que), chrysophanic acid, licochalcone $A$, luteolin, formononetin, honokiol, berberine $\mathrm{HCl}$, kaempferol, bergenin and synephrine (Fig. 1B and Table S1) with at least $15 \%$ higher cell proliferative ability than that of the vehicle control (DMSO) were identified. These ten compounds were further evaluated through a long-term treatment at different concentrations for 30 days (Fig. $1 A-C$ ). Que was selected due to its leading effects for further studies.

\section{Dose optimization of Que}

To identify the optimal concentration of Que in WS hMSCs (Fig. 2A), we first treated mid-passage (passage 5) WS hMSCs with different concentrations of Que for seven days. Then cell proliferative abilities were evaluated by MTS 


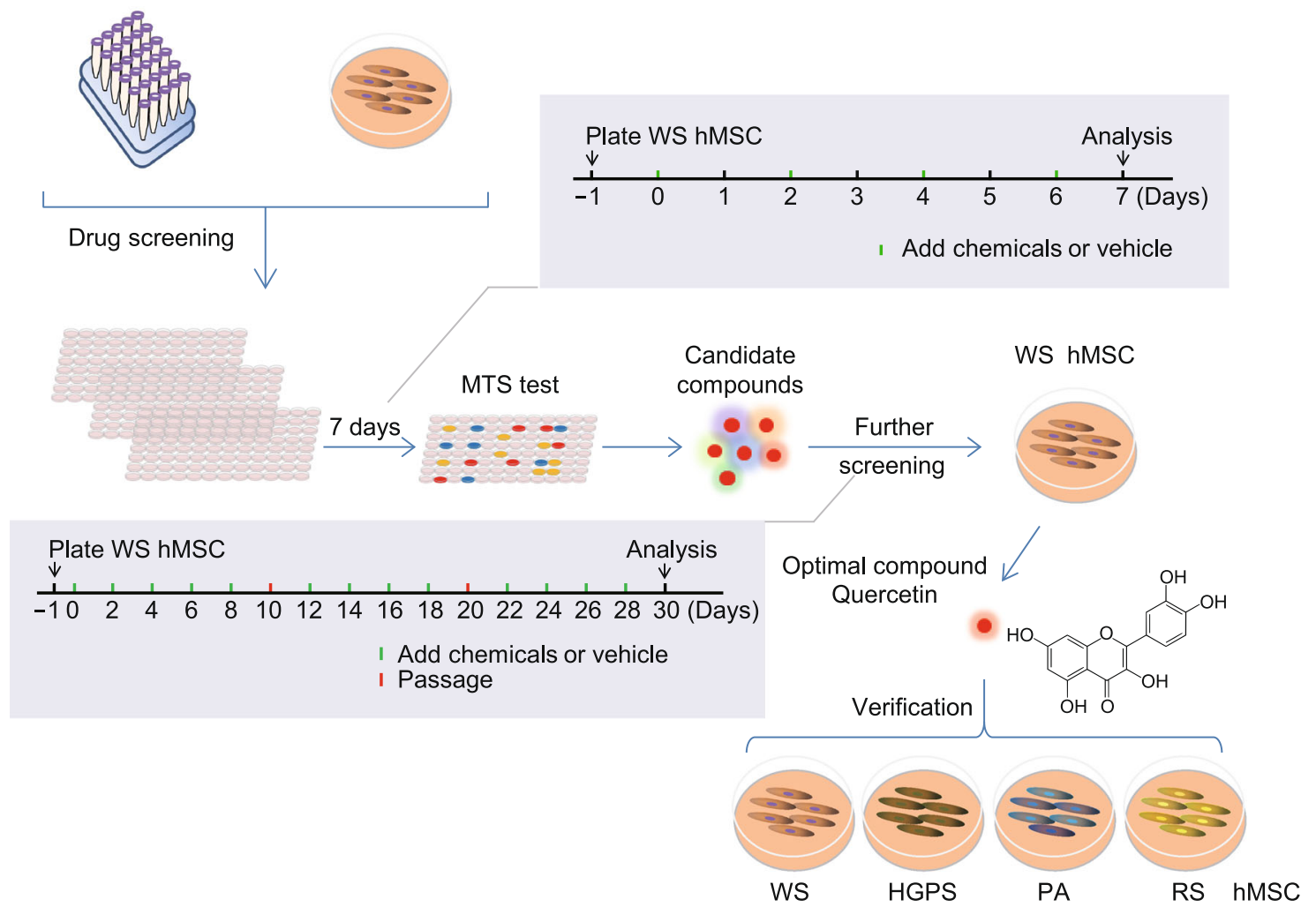

B

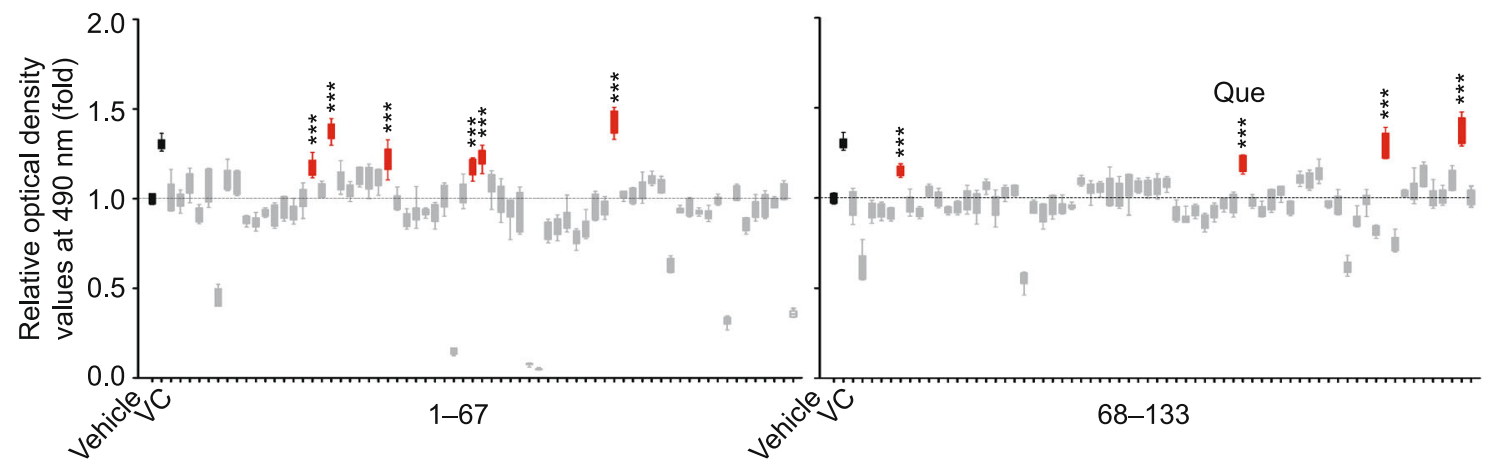

C

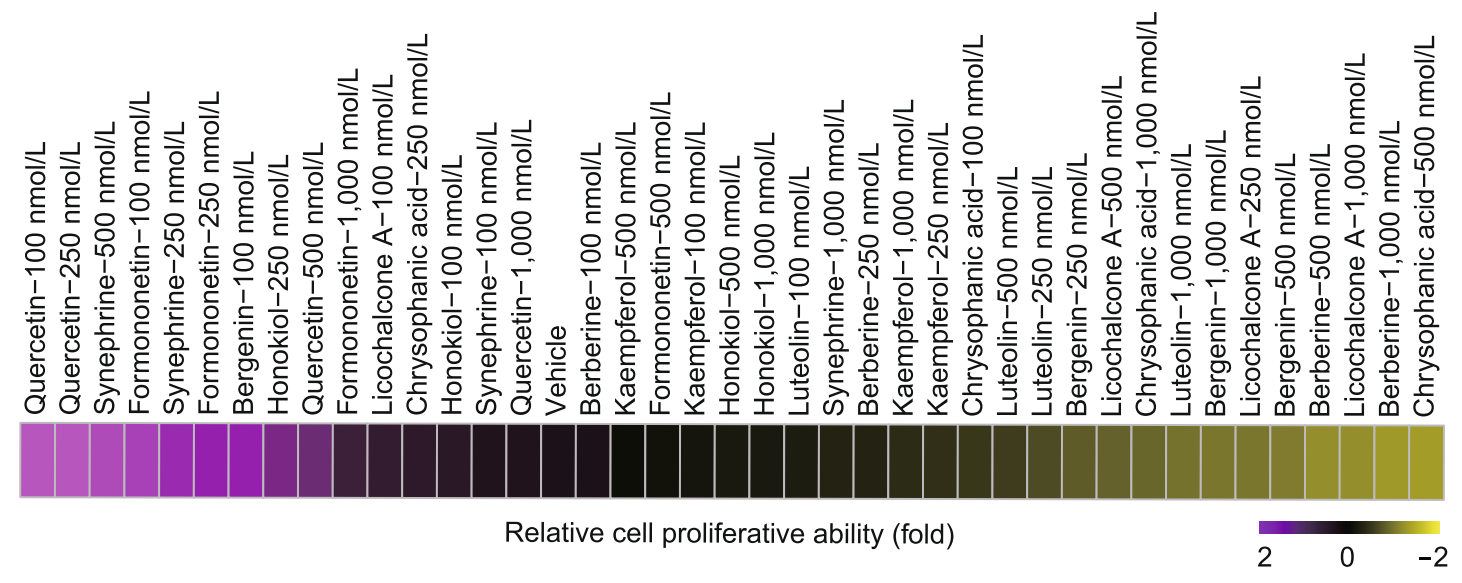




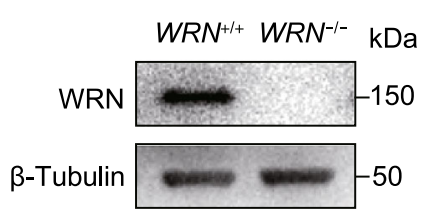

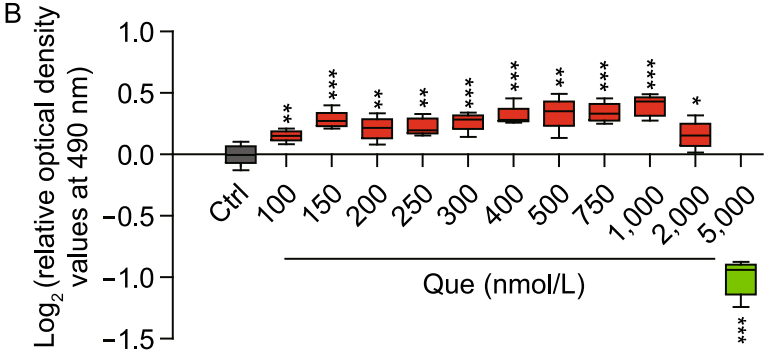
Que (nmol/L)

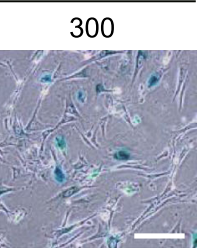

D
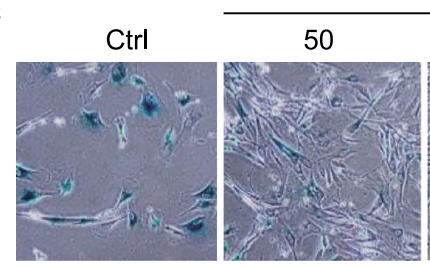

100
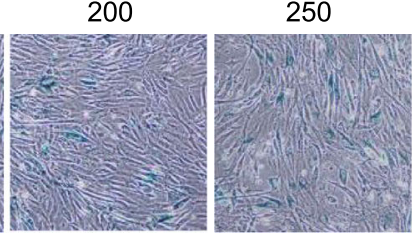

E
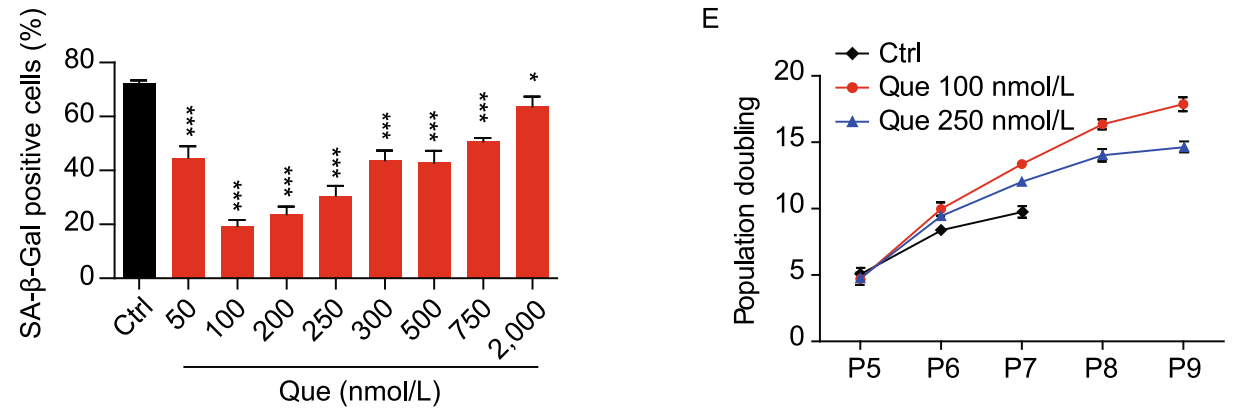

Figure 2. Identification of the optimal concentration of Que for alleviating aging and promoting proliferation of WS hMSCs. (A) Immunoblotting analysis of WRN protein in WT and WS hMSCs (passage 5). $\beta$-Tubulin was used as the loading control. (B) MTS assay of WS hMSCs (passage 5) after 7-day treatment of Que at a series of concentrations $(n=5) .{ }^{*} P<0.05$ vs. Ctrl; ${ }^{* *} P<0.01$ vs. Ctrl; ${ }^{* *} P<0.001$ vs. Ctrl. (C) Analysis of SA- $\beta$-Gal activity in vehicle- (Ctrl) and Que-treated WS hMSCs (passage 7). Representative images of SA- $\beta$-Gal staining. Scale bar, $100 \mu \mathrm{m}$. (D) Frequency of SA- $\beta-$ Gal positive cells $(n=3)$. ${ }^{*} P<0.05$ vs. Ctrl; ${ }^{* *} P<0.001$ vs. Ctrl. (E) Accumulative growth curve showing the population doubling of vehicle- and Que-treated hMSCs $(n=3)$.

assay, showing the beneficial effects of Que at concentrations ranging from $100 \mathrm{nmol} / \mathrm{L}$ to $2 \mu \mathrm{mol} / \mathrm{L}$ (Fig. 2B). Furthermore, we treated WS hMSCs (passage 5) with different concentrations of Que for a longer term (30 days). Assessment of senescence-associated- $\beta$-galactosidase (SA- $\beta$-Gal) and cellular proliferation ability of WS hMSCs at passage 7 identified the optimal concentration of Que at $100 \mathrm{nmol} / \mathrm{L}$ for mechanistic studies (Fig. 2C-E).

Que alleviated senescence and promoted cell selfrenewal and differentiation in WS hMSCs

Que-treated WS hMSCs were able to express hMSCspecific markers including CD73, CD90 and CD105 (Fig. 3A). Que alleviated the senescent phenotypes of latepassage WS hMSCs, evidenced by decreased senescent markers P16 and P21 (Fig. 3B), increased telomere length
(Fig. 3C), increased cell proliferative potential by Ki67 staining (Fig. 3D), and decreased DNA damage response markers $\mathrm{Y}-\mathrm{H} 2 \mathrm{AX}$ and 53BP1 (Fig. 3E). Reactive oxygen species (ROS) production (Labbé et al., 2010) (Fig. 3F), mRNA levels of proinflammatory cytokine IL-6 (Fig. 3G) and cell apoptosis (Fig. 3H) were each suppressed in Quetreated WS hMSCs. In addition, Que-treated WS hMSCs maintained the ability to differentiate towards multiple mesodermal lineages, with enhanced potentials towards osteogenesis and chondrogenesis (Fig. 4A). Furthermore, Que attenuated the in vivo decay of WS hMSCs implanted into the tibialis anterior muscles of nude mice (Fig. 4B) and enhanced the vasculogenic ability of WS hMSCs implanted into the fat pads of non-obese diabetic (NOD) scid mice (Fig. 4C). Taken together, these data suggest that Que alleviated cellular senescence and promoted self-renewal and differentiation abilities in WS hMSCs. 
A

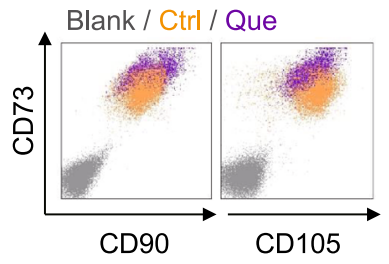

D

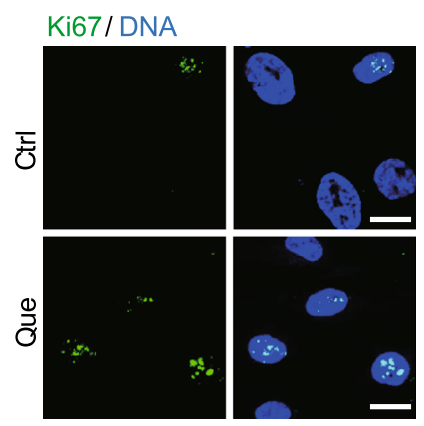

$\mathrm{F}$

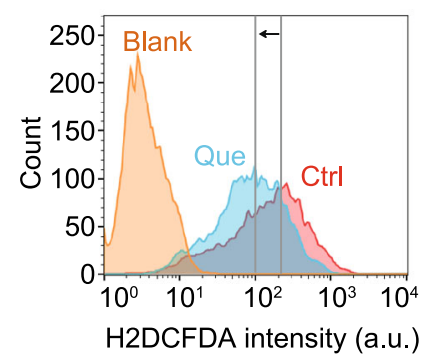

G
B
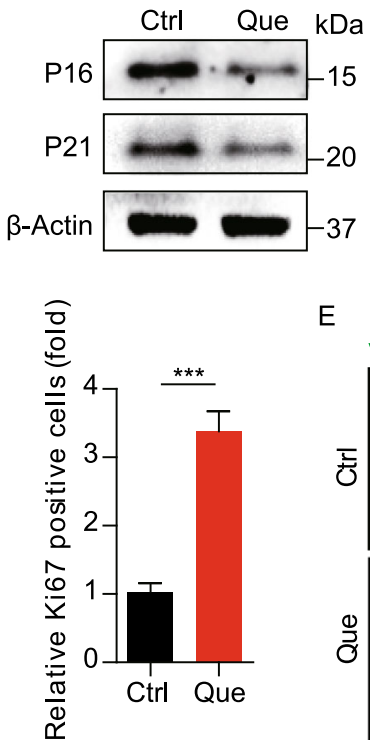

E

E
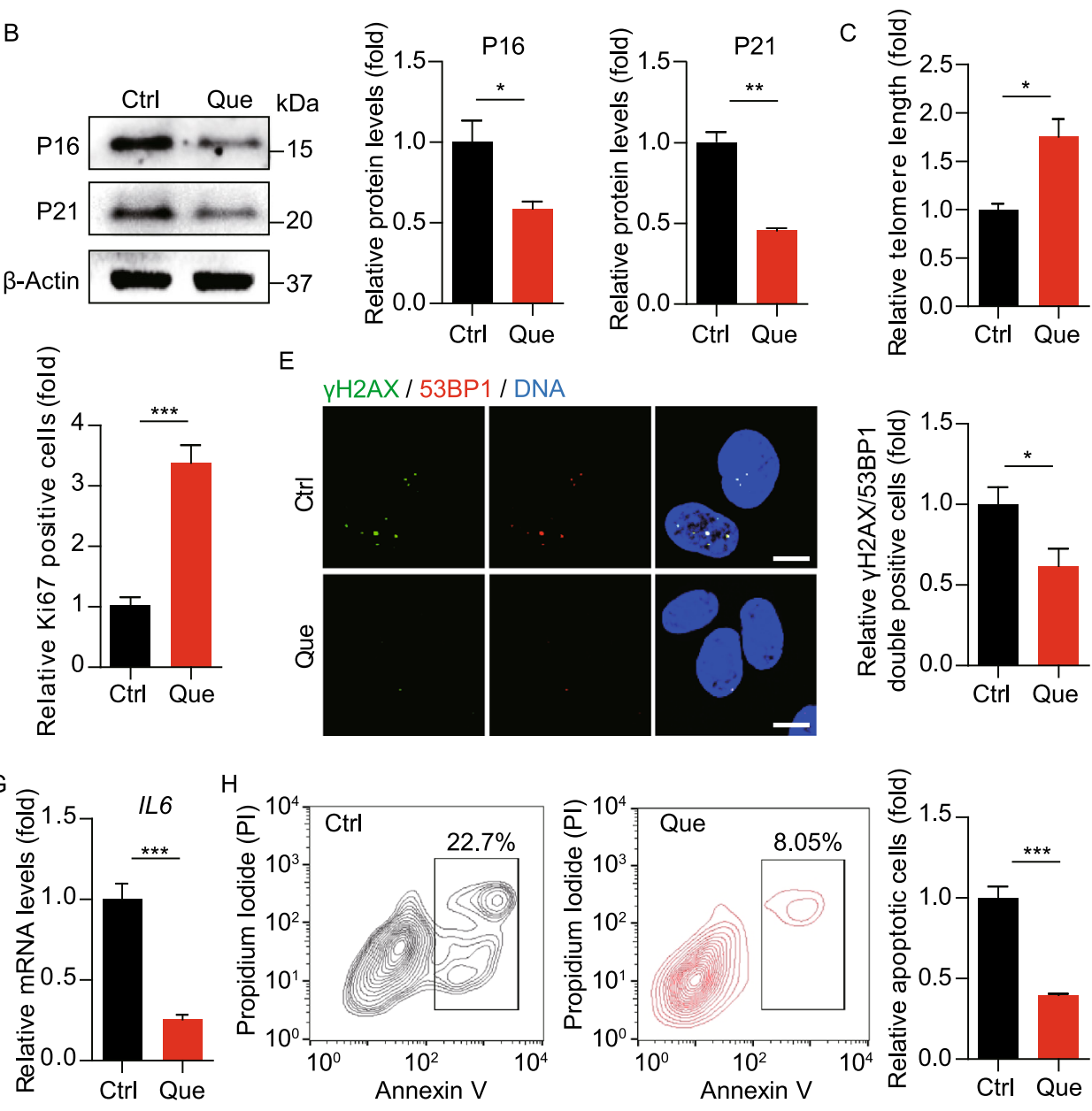

Figure 3. Que alleviated senescence and promoted self-renewal of WS hMSCs. (A) FACS analysis of hMSC-specific markers (CD73, CD90 and CD105) in vehicle- and Que-treated WS hMSCs. (B) Immunoblotting analysis of aging-related markers P16 and P21 in vehicle- and Que-treated WS hMSCs (passage 7). $\beta$-Actin was used as the loading control. Data are shown as mean \pm SEM $(n=3) .{ }^{*} P<0.05$ vs. Ctrl; ${ }^{*} P<0.01$ vs. Ctrl. (C) Quantitative PCR analysis of telomere length of vehicle- and Que-treated WS hMSCs (passage 7). Data are shown as mean $\pm \operatorname{SEM}(n=3)$. ${ }^{*} P<0.05$ vs. Ctrl. (D) Immunostaining of Ki67 in vehicle- and Que-treated WS hMSCs (passage 7), Scale bar, $25 \mu \mathrm{m}$. Data are shown as mean \pm SEM (cell number $\geq 300$ ). ${ }^{* * *} P<0.001$ vs. Ctrl. (E) Immunostaining of $\mathrm{Y}-\mathrm{H} 2 \mathrm{AX}$ and 53BP1 in vehicle- and Que-treated WS hMSCs (passage 7), Scale bar, $10 \mu \mathrm{m}$. The percentage of $\mathrm{Y}-\mathrm{H} 2 \mathrm{AX} / 53 \mathrm{BP} 1$-double positive cells are shown as mean $\pm \mathrm{SEM}$ (cell number $\geq 300$ ). ${ }^{*} P<0.05$ vs. Ctrl. (F) FACS measurement of reactive oxygen species (ROS) by H2DCFDA in vehicle- and Que-treated WS hMSCs (passage 7). a.u., arbitrary unit. (G) RT-qPCR analysis of IL6 mRNA levels in vehicle- and Que-treated WS hMSCs (passage 7). Data are shown as mean \pm SEM $(n=3)$. ${ }^{* *} P<$ 0.001 vs. Ctrl. (H) Cell apoptosis assay in vehicle- and Que-treated WS hMSCs (passage 7). Quantitative data on the right are presented as mean $\pm \operatorname{SEM}(n=3)$. ${ }^{* * *} P<0.001$ vs. Ctrl.

\section{Que restored the heterochromatin architecture in WS hMSCs}

Because Werner syndrome is caused by mutations in WRN gene that encodes a RecQ DNA helicase important to DNA replication and chromatin maintenance (Li et al., 2016b; Wu et al., 2018; Yu et al., 1996; Zhang et al., 2015), genomic instability and heterochromatin disorganization manifest in WS pathogenesis (Li et al., 2016b; Murfuni et al., 2012; Ren et al., 2017a; Ren et al., 2011; Seki et al., 2008; Shamanna et al., 2017; Wu et al., 2018; Zhang et al., 2015). Upon Que treatment, the transcription of pericentromeric repetitive sequences including $\alpha$-satellite ( $\alpha$-Sat) and satellite 2 (Sat2) were downregulated, whereas nuclear envelope proteins Lamin B1 and LAP2 $\beta$ were transcriptionally upregulated (Fig. 5A). Likewise, the protein levels of LAP2 $\beta$ and heterochromatin marker HP1y were also increased (Fig. 5B and 5C). Lastly, direct visualization of nuclear structure by transmission electron microscope (TEM) revealed 

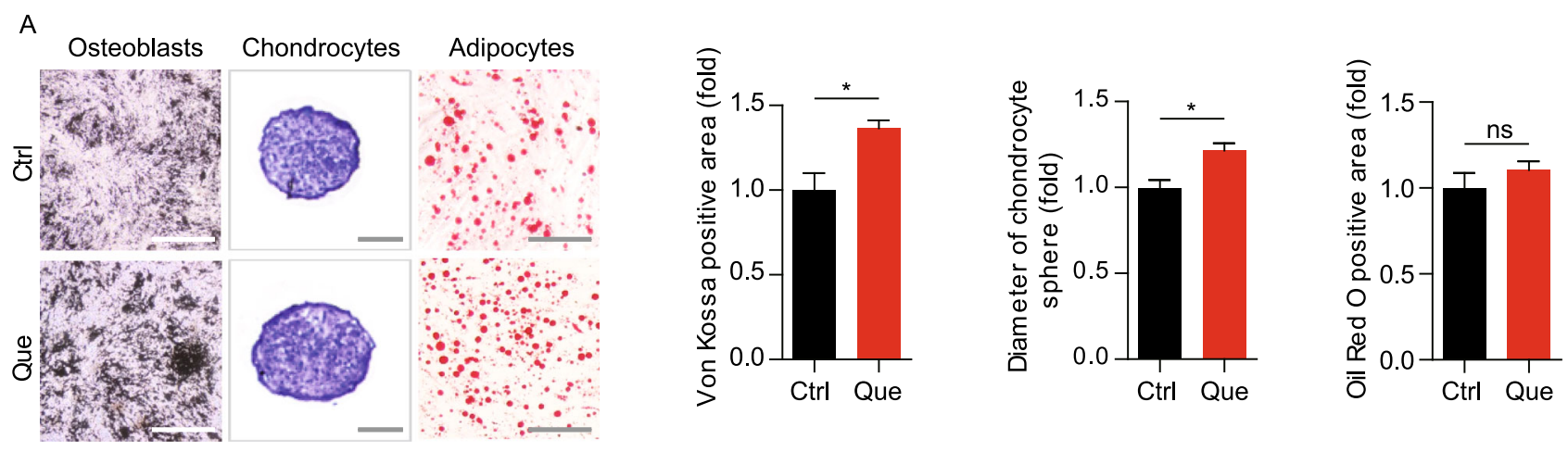

B
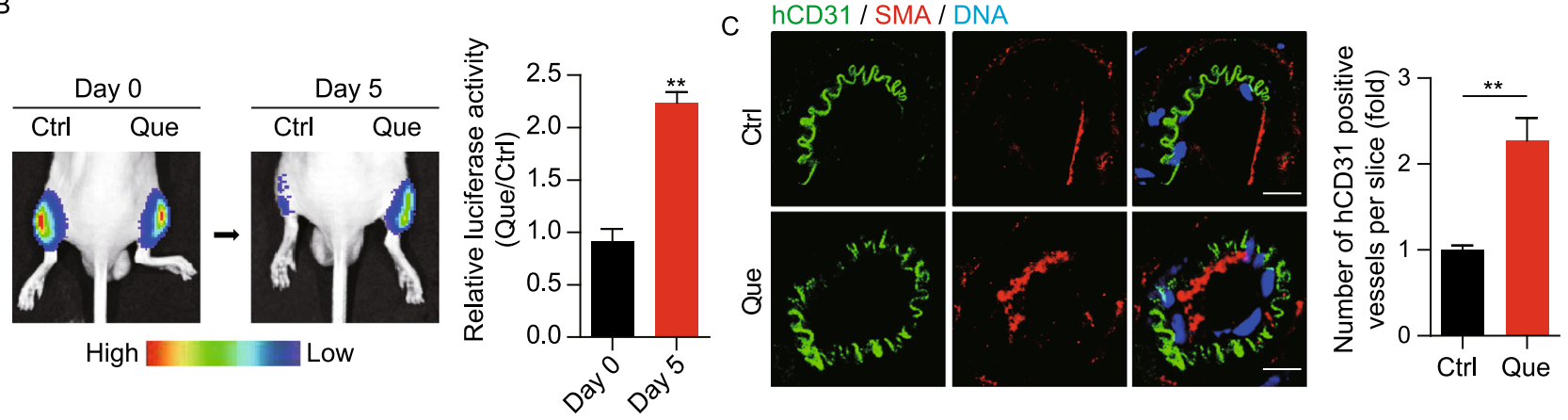

Figure 4. Que promoted cellular differentiation and vasculogenesis of WS hMSCs. (A) Characterization of osteogenesis, chondrogenesis and adipogenesis potentials of vehicle- and Que-treated WS hMSCs (passage 7) stained by Von Kossa ( $n=3$ independent experiments), toluidine blue $\mathrm{O}(n=15$ spheres) and oil red $\mathrm{O}(n=3$ independent experiments), respectively. Data are presented as mean \pm SEM. ${ }^{*} P<0.05$ vs. Ctrl; ns, not significant. Scale bar, $100 \mu \mathrm{m}$. (B) In vivo hMSC implantation assay with vehicleand Que-treated WS hMSCs. Quantitative data on the right are presented as mean \pm SEM. ${ }^{* *} P<0.01$ vs. Ctrl $(n=6)$. (C) Fat pad transplantation with vehicle- and Que-treated WS hMSCs (passage 7). Left: representative immunofluorescent images showing neovascularization; right: the number of hCD31-positive vessels calculated based on 20 slices from inconsecutive frozen sections from six mice each group. ${ }^{*} P<0.01$ vs. Ctrl. Scale bar, $10 \mu \mathrm{m}$.

heterochromatin loss reduced from $73.9 \%$ to $37.0 \%$ in WS hMSCs upon Que treatment (Fig. 5D). Taken together, these data suggest that Que repressed heterochromatin disorganization in premature aging hMSCs.

\section{Transcriptome analysis revealed rejuvenation of WS hMSCs by Que}

To uncover the molecular mechanisms by which Que rejuvenated WS hMSCs, we performed genome-wide RNA sequencing (RNA-seq). The correlation coefficients between replicates were 0.9999 in vehicle- (Ctrl) and Que-treated WS hMSCs, indicating that the RNA-seq data were of high reproducibility (Fig. 6A). A total of 486 upregulated genes and 294 downregulated genes were obtained in Que-treated WS hMSCs relative to Ctrl $\left(\mid \log _{1.5}\right.$ (fold change) $\mid>1$, adjust $P$ value (padj) $<0.05$ ) (Fig. 6B, 6C, Tables S2 and S3). Protein-protein interactions (PPI) network of differentially expressed genes was drawn based on the STRING database (Fig. 6D). Gene Ontology (GO) analysis revealed that the upregulated genes in Que-treated WS hMSCs were enriched in biological processes including cell cycle, cell division, chromosome segregation, and cell proliferation (Fig. 6E and 6F). Consistently, gene set enrichment analysis (GSEA) data revealed that Que-treated WS hMSCs were enriched in GO terms including cell cycle, condensed chromosome, as well as cellular response to oxygen, UV and vitamin (Fig. $6 \mathrm{~F}$ and $6 \mathrm{G}$ ). On the contrary, vehicle-treated WS hMSCs were enriched in apoptosis pathway (Fig. 6G). The anti-apoptotic function of Que was further evidenced by the increased transcriptional levels of typical anti-apoptotic genes including genes in BCL2, IAP and $A K T$ families (Fig. 6F), consistent with the aforementioned results showing decreased cell apoptosis in Que-treated WS hMSCs.

To investigate whether Que was directly involved in the regulation of aging biological process, we checked the transcriptional levels of aging and longevity-associated genes annotated by the Human Ageing Genomic Resources (HAGR) database. Gene expression analysis showed that aging and longevity-associated genes were upregulated $(P<$ 0.0001 ) upon Que treatment (Fig. 7A-C). Of note, a series of anti-oxidative genes including GSTP1, GSR and GSTM1 
A

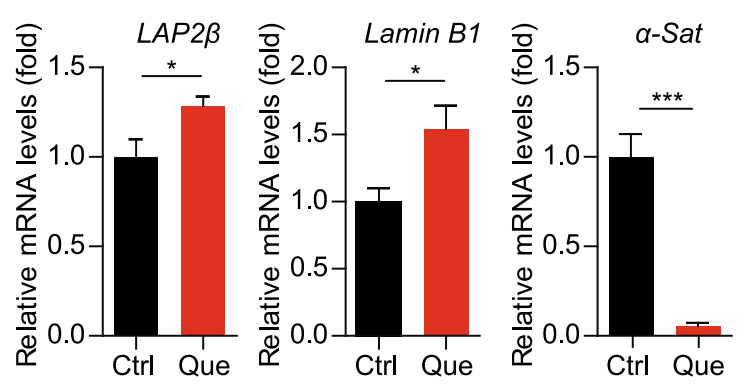

B

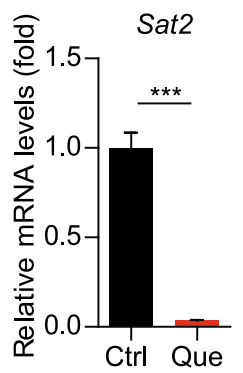

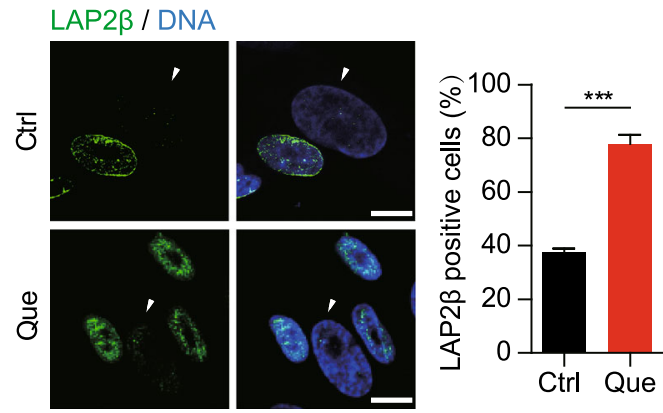
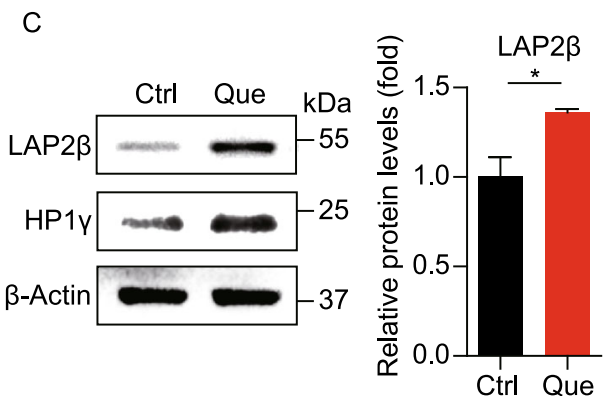
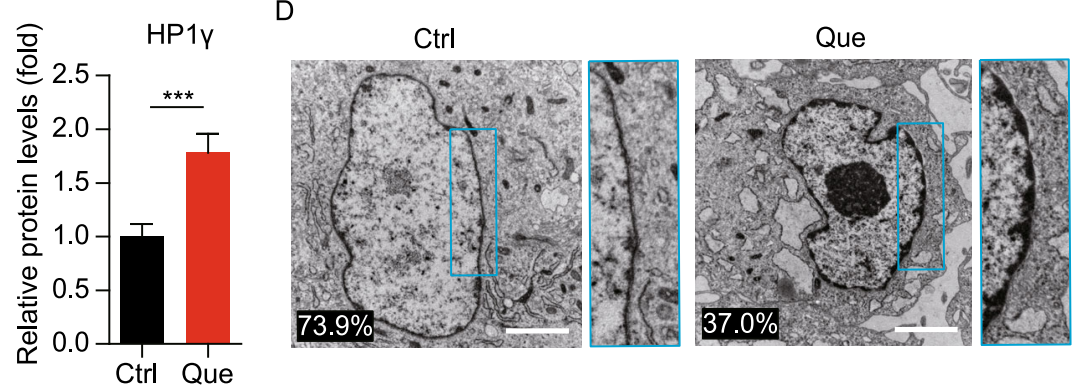

Figure 5. Que rejuvenated epigenetic features of WS hMSCs. (A) RT-qPCR analysis of LAP2 $\beta$, Lamin B1, $\alpha$-Sat and Sat2 mRNA levels in vehicle- and Que-treated WS hMSCs (passage 7). Data are shown as mean \pm SEM $(n=3)$. ${ }^{*} P<0.05$ vs. Ctrl; ${ }^{* \star *} P<0.001$ vs. Ctrl. (B) Immunostaining of LAP2 $\beta$ in vehicle- and Que-treated WS hMSCs. White arrows indicate LAP2 $2 \beta$-negative nuclei. Quantitative data on the right are shown as mean \pm SEM (cell number $\geq 300$ ). ${ }^{* \star *} P<0.001$ vs. Ctrl. Scale bar, $10 \mu \mathrm{m}$. (C) Immunoblotting analysis of LAP2 $\beta$ and HP1Y in vehicle- and Que-treated WS hMSCs (passage 7). $\beta$-Actin was used as the loading control. Quantitative data are shown as mean $\pm \operatorname{SEM}(n=3)$. ${ }^{*} P<0.05$ vs. Ctrl; ${ }^{\star * *} P<0.001$ vs. Ctrl. (D) Visualization of heterochromatin architecture of vehicle- and Que-treated WS hMSCs (passage 7) by transmission electron microscope (TEM). Highmagnification views of heterochromatin around nuclear envelope are shown on the right. The percentage of cells with sparse heterochromatin at nuclear periphery is indicated at the lower left corner of each representative image. Cell number $>100$. Scale bar, $2 \mu \mathrm{m}$.

were upregulated (Fig. 7C). Given that all these genes were NRF2 responsive genes, we tested whether the anti-oxidative function of Que was NRF2-dependent by evaluating the expression levels of NRF2 target genes. The results showed that NRF2 target genes were upregulated $(P<0.0001)$ upon Que treatment (Fig. 7D). The increased expression levels of GSTP1, GSR, SOD1 and SOD2 in WS hMSCs upon Que treatment was reconfirmed by RT-qPCR analysis (Fig. 7E). Taken together, these data suggest that Que rejuvenated WS hMSCs via the regulation of multiple cellular processes including promoting cell cycle, condensing chromosome, and enhancing anti-oxidation.

\section{Transcriptomics comparison of the geroprotective effects of Que and VC in WS hMSCs}

We have previously reported that Vitamin C (VC) is a rejuvenating factor for WS hMSCs by altering the expression of genes involved in chromatin condensation, cell cycle regulation, DNA replication, and DNA damage repair pathways (Li et al., 2016b). Here, we performed a conjoint analysis of RNA-seq data for Que- and VC-treated WS hMSCs to compare and contrast the underlying mechanisms of their geroprotective effects. Firstly, the upregulated and downregulated genes in WS hMSCs compared to WT hMSCs were reversed by either Que or VC treatment (Fig. $8 \mathrm{~A}$ and $8 \mathrm{~B}$ ). We further analyzed the upregulated genes in Que- and VC-treated WS hMSCs. Among these genes, 333 were specific to Que treatment and 721 to VC treatment, with 153 to both Que and VC treatment (Fig. 8C). Notably, the commonly upregulated genes were enriched in biological process GO terms related to cell cycle, chromatin condensation and anti-oxidation, whereas Que-specific upregulated genes were mostly enriched in response to stimulus and cell homeostasis and the VC-specific ones in DNA replication, DNA repair and telomere maintenance (Fig. 8D). Thus, these data reveal the major commonalities in the mechanisms by which Que and VC alleviated aging phenotypes in WS hMSCs.

Que attenuated senescence in Hutchinson-Gilford progeria syndrome hMSCs

Besides WS, Hutchinson-Gilford progeria syndrome (HGPS) hMSCs are another premature senescent stem cell model we recently established by introducing heterozygous 
A
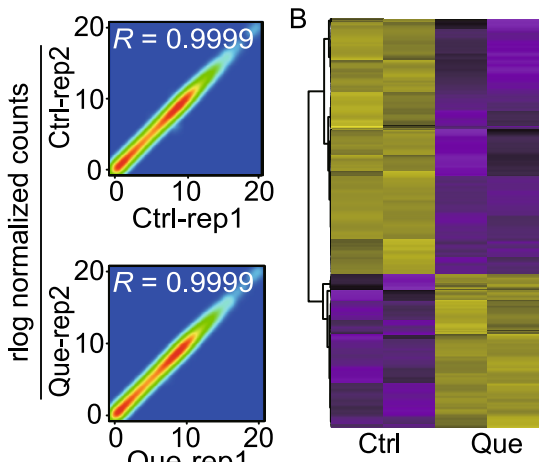

rlog normalized counts

E

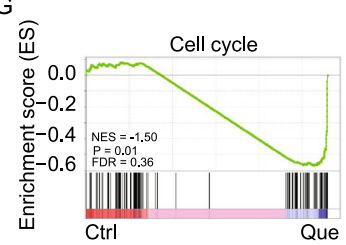

焉 Condensed chromosome

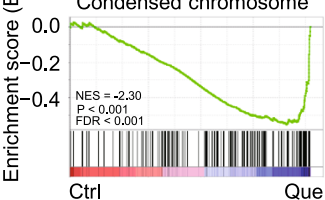

足 Cellular response to oxygen

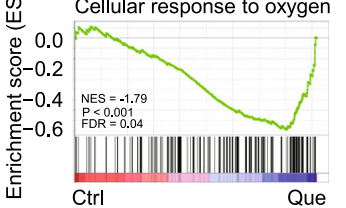

1
0
-1

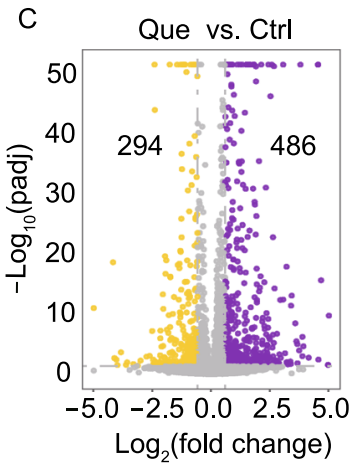

F Cell proliferation
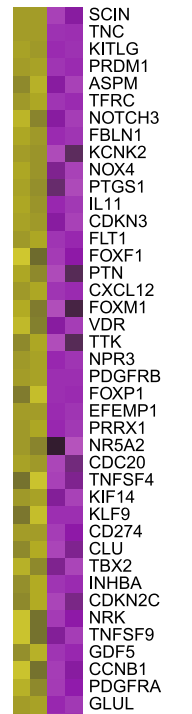

Cell cycle
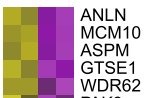
WDR62 PAK3 NPX2
TIF4A CDNN3 NCAPG FOXM1 TTK LMNB1 PDGFRB SMC4
CENPA CDC2O STMN1
NEK2 NEK2
CENPF KIF 14 $\mathrm{NCAPH}$ INHBA ZWIN CDKN2C DKMYT1 TUBA4A CCNB CDCA8 KIF23 KIF11 CEP55 SIK1 $\mathrm{KIIF} 2 \mathrm{C}$

NUF2 CCNA2
NCAPG2 NEK7 TCF7L1 CCSAP BTG3 BUB1 CCNB2 RACGAP1 MAD2L1 PTTG1
PIK1 PLK1 RRM1 BUB1 CDK1
RRM2 RRM2 SLFN11 UBE2C PLCB1
IQGAP3 $\mathrm{NES}=1.07$
$P=0.30$
$\mathrm{FDR}=1.00$

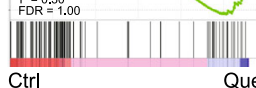
Que

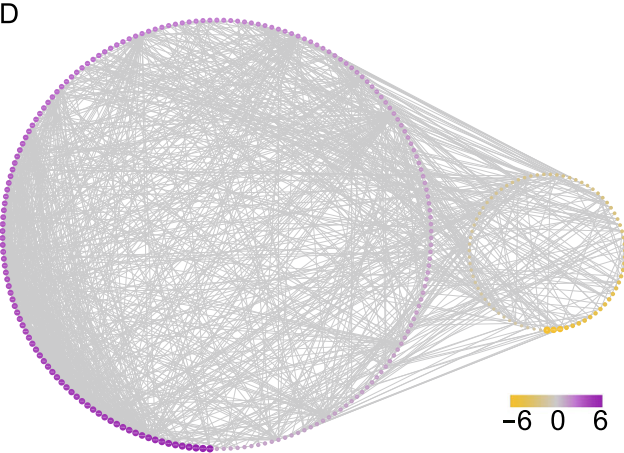
division

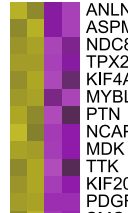

ANLN
ASPM
NDC8
TPX2
KIF4A
MYBL2
PTN
NCAP
MDK
TTK
KIF20A
PDGF

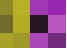
ECT2
CDC20

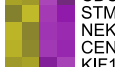

KIF 14
NCAPH
ZWINT
CIT

CIT
TUBA1B
DLGAP5
PKMYT1 PKMYT1
MIS18BP1 MIS18B
TOP2A
CCNB1 CCNA1
CDCA8 NUSAP
KIF23 KIF11 FGF5
CENPE
KIF2C
NUF2
CCNA2 CCNA2 2 MKI67
VEGFC

VEGFC
NEK7
TCF7L1
PELO
CCSAP

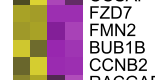

CCNB2
RACGAP1

MAD2L1
PTTG1
PLK1
PBK
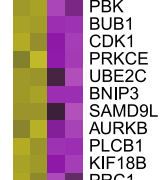

Ctrl Que

apoptosis

${ }_{\mathrm{BCL} 2 \mathrm{~A} 1}^{\mathrm{BCL2}}$

BCL2L1
BCLL11
BCL2L12
BC2L12

BCL212

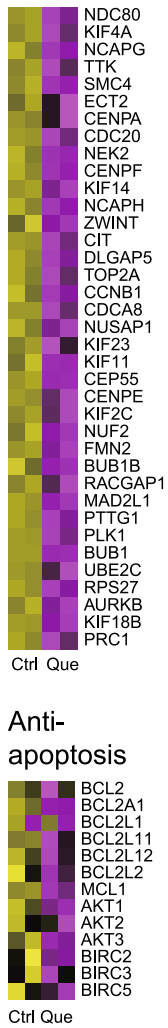

Ctrl Que

Cellular response to oxygen levels
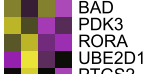

GNB

PPP1R15A
DNMT3B

SLC9A

RBX1
HIF1A
SLCBA

ANGPT4
SUU39H1
EEF $2 \mathrm{~K}$
TCE

SFRP1
SNIP3L
BNIL

CAV

CAA
UBED3
PPARD
VEGFA
VEGEA
CFEBA

CPEB4
FAM162A
EPS1

EPAS1
EGR1
TWIS1
PLAU 1
HLE 3 A

HIA3A
HP1BP3
EGLN3
HEL 325

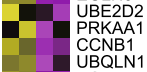

EGLN

MMAP
PMT1
AKT1

ARNT
RPS27A
CCNA2
NONA

CCNA2
NOTCH1
ANKRD1

PYK1
POK1
TCEB

FMN2
BACH
BACH

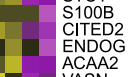

ENDOG
ACAA2
VAAS

BDSR
ADRB2
PRKCE
BCL2

BNP2A4
SLC8A1

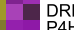

\begin{tabular}{l} 
EIF4EBP1 \\
ERO1L \\
LPAR1 \\
\hline
\end{tabular}

Ctrl Que

1
0
-1 
4 Figure 6. Gene expression profile analysis of vehicle- and Que-treated WS hMSCs. (A) Scatter plots showing the correlation between replicates of vehicle- (Ctrl) and Que-treated WS hMSCs. (B) Heatmap illustrating differentially expressed genes in Que-treated WS hMSCs compared to Ctrl (passage 7). (C) Volcano plot showing the number of upregulated and downregulated genes upon Que treatment. (D) Interaction network showing protein-protein interactions (PPI) of differentially expressed genes with $\mid \log _{2}$ (fold change) $\mid>1$ and interaction score $>0.9$ based on the STRING database. Purple indicates upregulation and yellow indicates downregulation; node size indicates the degree of upregulation and downregulation. (E) GO enrichment analysis of upregulated genes in Que-treated hMSCs. (F) Heatmaps showing the transcriptional levels of genes enriched in various gene terms. (G) Gene set enrichment analysis (GSEA) plots showing representative gene terms related to the functions of Que. The plots were based on the results from KEGG analysis for the cell cycle and apoptosis pathways and $\mathrm{GO}$ analysis for the condensed chromosome and cellular response to oxygen, vitamin and UV.

LMNA ${ }^{\mathrm{G608G/+}}$ mutation into human embryonic stem cells ( $\mathrm{hESCs}$ ) followed by directed differentiation into hMSCs (Kubben et al., 2016; Wu et al., 2018). DNA sequencing confirmed the presence of heterozygous mutation of LMNA ${ }^{\mathrm{G} 608 \mathrm{G} /+}$ in HGPS hMSCs (Fig. 9A). The mRNA level and immunostaining intensity of progerin, which is the resultant truncated protein product of mutated $L M N A$, were decreased in Que-, VC- and VC plus Que-treated HGPS hMSCs (Fig. 9B and 9C). The alleviation of cellular senescence by Que, VC, or VC plus Que in HGPS hMSCs was further evidenced by decreased population doubling time (Fig. 9D), decreased SA- $\beta$-Gal positivity (Fig. 9E), and increased clonal expansion and proliferative ability (Fig. 9F$\mathrm{H})$. In addition, the percentage of abnormal nuclei, a cellular hallmark of HGPS, decreased in HGPS hMSCs upon Que treatment (Fig. 9I). Taken together, these data indicate that Que attenuated accelerated senescence in HGPS hMSCs.

\section{Que ameliorated senescent phenotypes}

in physiological-aging hMSCs

We further examined the geroprotective effects of Que in physiological-aging (PA) wild-type hMSCs from a 56-year individual and replicative-senescent (RS) wild-type hMSCs differentiated from hESCs. Decreased population doubling time and SA- $\beta$-Gal positivity revealed enhanced self-renewal and suppressed senescence in PA and RS hMSCs upon Que treatment, respectively (Fig. 10A-D). In addition, cell apoptosis was alleviated in Que-treated RS hMSCs (Fig. 10E). These results suggest that besides progeria cells, Que also exerted geroprotective effects against physiological senescence in hMSCs.

\section{DISCUSSION}

Discovery of compounds that attenuate senescent phenotypes can lead to therapeutic advances that ameliorate aging-related pathologies (Chang et al., 2016; Kreienkamp et al., 2016; Li et al., 2016b). Here, by employing a platform with premature aging Werner syndrome (WS) hMSCs for the screening of effective compounds against cellular senescent phenotypes, we have identified ten candidate natural products, including quercetin dihydrate, chrysophanic acid, licochalcone A, luteolin, formononetin, honokiol, berberine $\mathrm{HCl}$, kaempferol, bergenin and synephrine. Except Que and berberine $\mathrm{HCl}$ (Xu et al., 2017), the other eight compounds have never been implicated in premature aging-related studies, thus greatly broadening our view of geroprotective drug candidates. Further investigation revealed the mechanisms by which the top hit, Que exhibited beneficial effects in attenuating hMSC senescence: (1) Que alleviated senescence by promoting stem cell self-renewal and restoring the heterochromatin architecture in WS hMSCs; (2) transcriptome analysis supported that Que improved the "healthspan" of WS hMSCs; (3) Que and VC exerted geroprotective effects through overlapping mechanisms; (4) Que also alleviated cellular senescent phenotypes in HGPS and physiological-aging hMSCs (Fig. 11). For the first time, Que was identified as a geroprotective agent against both premature and physiological human aging, supporting the role of Que as a novel therapeutic option for treating premature aging and promoting healthy aging. Additionally, the generalized effectiveness of Que verifies the efficacy of WS hMSCs as a powerful model for geroprotective drug screening.

Que is a polyphenol derived from plants. In C. elegans, Que extends lifespan by $15 \%$ due to its antioxidant capacity indirectly via the activation of DAF-16 (homologue to mammalian FoxO proteins) (Kampkötter et al., 2008). Que is also reported to rejuvenate senescent primary human fibroblasts (Chondrogianni et al., 2010). Prior to our study, the effect of Que has not been evaluated in progeroid syndromes such as WS and HGPS. For the first time, we uncovered a geroprotective role of Que in human accelerated aging hMSCs. Que-treated WS hMSCs demonstrated improved differentiation potentials into osteoblasts and chondrocytes and enhanced in vivo vasculogenesis ability, implying potential therapeutic promises of Que with regard to symptoms such as osteoporosis and atherosclerosis in WS and HGPS patients. Of particular, we observed that Que effectively decreased progerin in HGPS hMSCs. Thus, our studies support Que as a potential therapeutic agent for treating WS and HGPS in clinic (Harhouri et al., 2018). Still, the in vivo effects of Que on rodents or non-human primates need further investigation.

The effects of high-dose Que have been previously evaluated in human cells and mice. The combination of dasatinib and high-dose of Que $(50 \mathrm{mg} / \mathrm{kg})$ effectively eliminates senescent cells via induced apoptosis and thus alleviated senescence-related phenotypes in mice (Zhu et al., 2015). In 
A

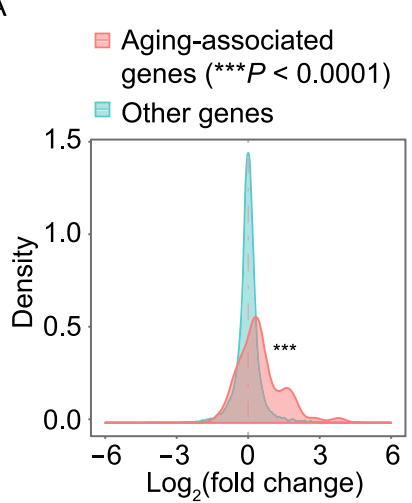

B
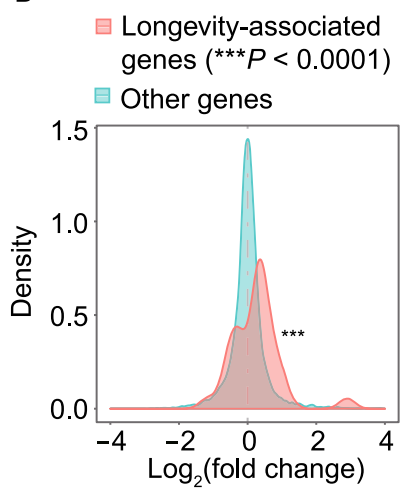

C
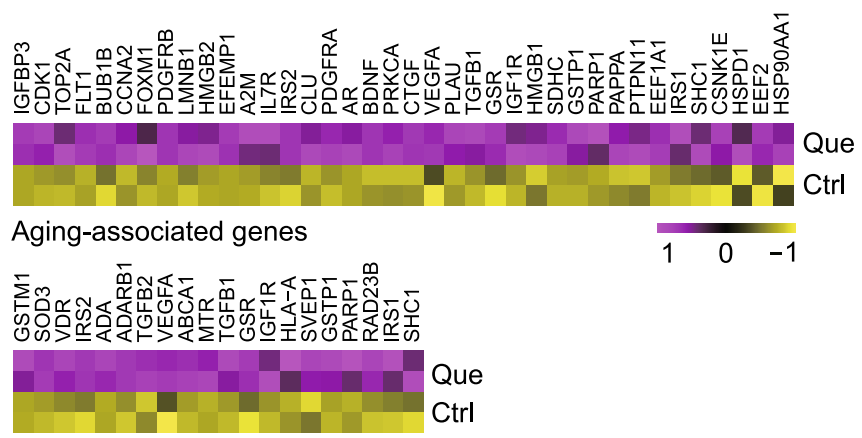

Longevity-associated genes

D

$$
\begin{aligned}
& \text { NRF2 target genes } \quad \text { E } \\
& \left({ }^{* * *} P<0.0001\right)
\end{aligned}
$$

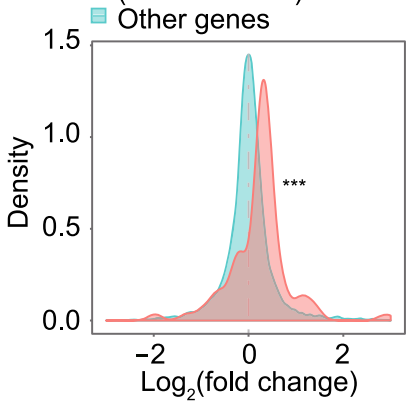

Ctrl Que

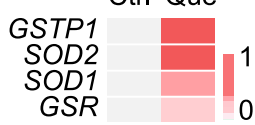

Figure 7. Gene expression analysis revealed the regulation of aging/longevity-related genes, and NRF2 target genes upon Que treatment. (A) Density plot showing $\log _{2}$ (fold change) of mRNA expression levels between Que-treated and vehicle-treated (Ctrl) WS hMSCs for aging-associated genes. A rightward shift $\left.{ }^{* * *} P<0.0001\right)$ indicates increased frequency of genes upregulated upon Que treatment. (B) Density plot showing $\log _{2}$ (fold change) of mRNA expression levels between Que-treated and Ctrl WS hMSCs for longevity-associated genes. (C) Heatmap showing upregulated genes associated with aging and longevity upon Que treatment. (D) Density plot showing $\log _{2}$ (fold change) of mRNA expression levels between Que-treated and Ctrl WS hMSCs for NRF2 target genes. (E) RT-qPCR analysis of the transcriptional levels of NRF2 target genes in vehicle- and Que-treated WS hMSCs $(n=3)$ (passage 7).

non-alcoholic fatty liver disease (NAFLD) mice, the combination of dasatinib and Que $(20 \mathrm{mg} / \mathrm{kg})$ eliminates senescent cells for the reduction of overall hepatic steatosis (Ogrodnik et al., 2017). In senescent human preadipocytes and umbilical vein endothelial cells (HUVECs), high-dose Que (optimal concentrations at 10 and $20 \mu \mathrm{mol} / \mathrm{L}$, respectively) induces apoptotic cell death. By contrast, we used Que at $100 \mathrm{nmol} / \mathrm{L}$ in WS, HGPS, physiological-aging hMSCs, which effectively alleviated cellular senescence without inducing cell death, indicative of a much safer therapeutic dose potentially applicable to stimulating hMSC activity.

To further determine the molecular mechanisms of Que, we performed RNA-seq analysis and revealed that the upregulated genes in Que-treated WS hMSCs were primarily 


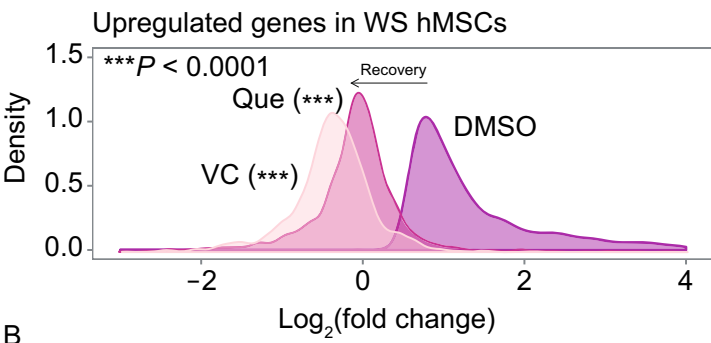

B

Downregulated genes in WS hMSCs

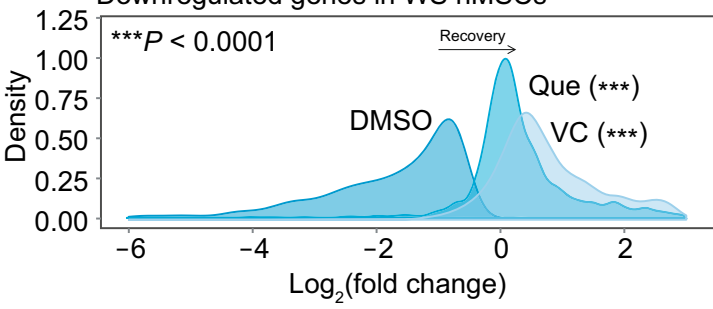

C

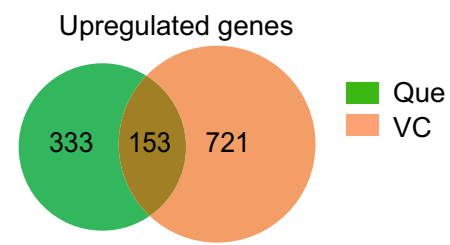

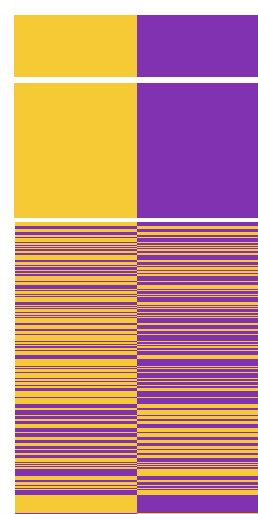

Ctrl

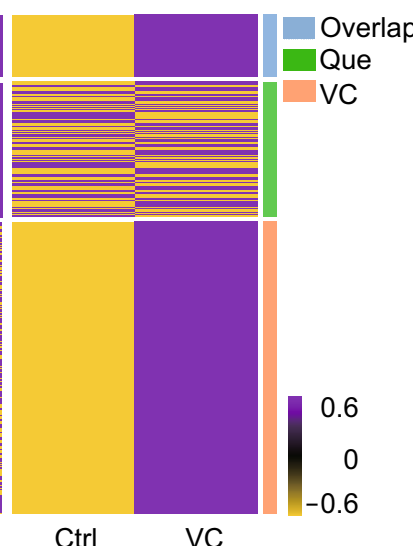

$\mathrm{D}$

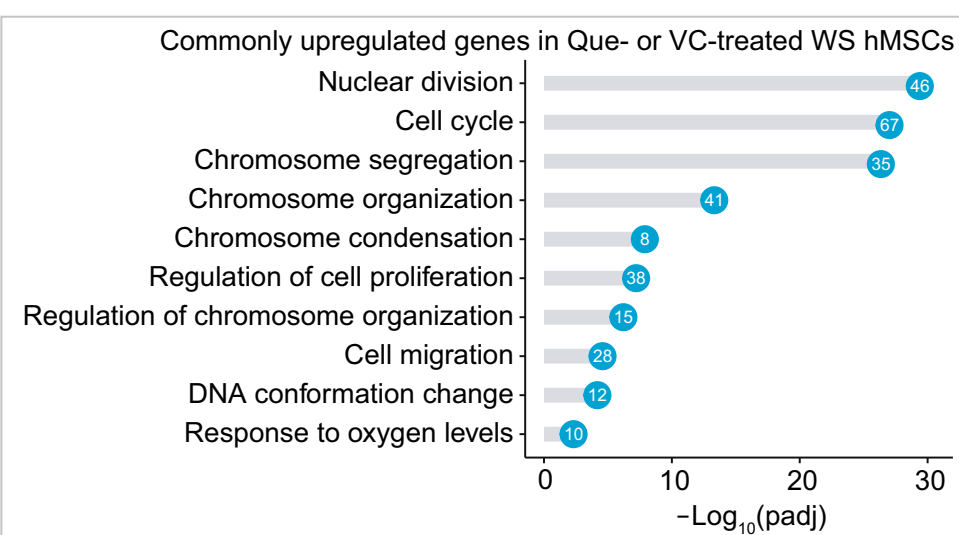

Specifically upregulated genes in Que-treated WS hMSCs Positive regulation of cell proliferation -

Response to oxygen levels.

Positive regulation of cell migration

Response to endogenous stimulus Angiogenesis

NAD metabolic process Cellular homeostasis

Cellular response to vitamin Cellular response to external stimulus Regulation of cytokine production Apoptotic cell clearance

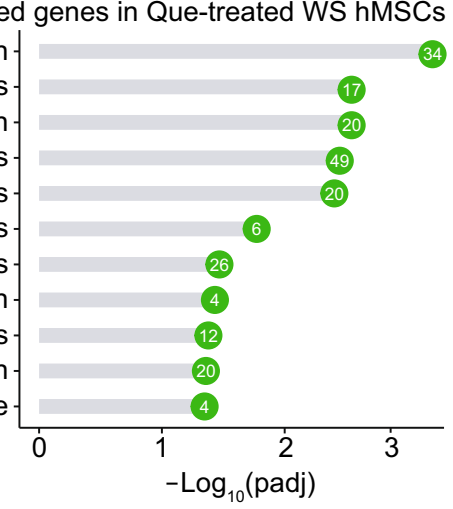

Specifically upregulated genes in VC-treated WS hMSCs

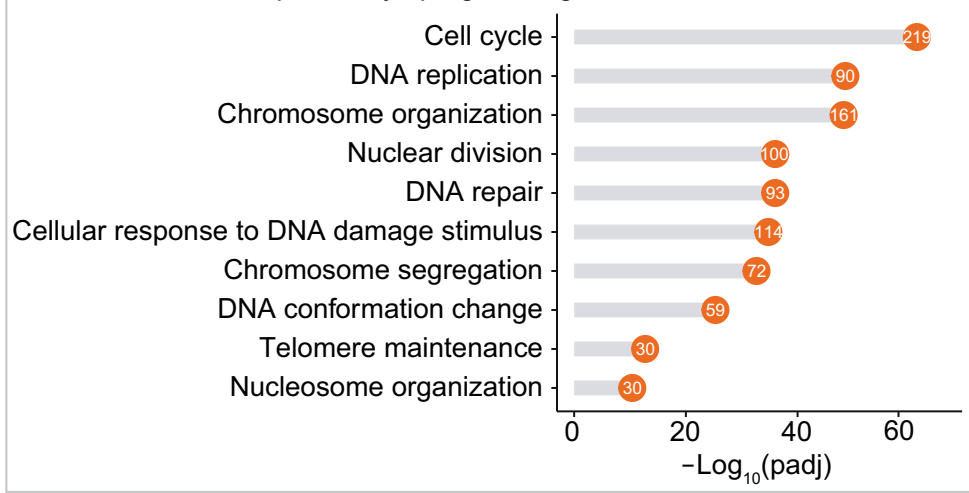

Figure 8. Comparative analysis of the RNA-seq data for Que- and VC-treated WS hMSCs. (A) Density plot showing upregulated genes in WS hMSCs restored upon Que or VC treatment. (B) Density plot showing downregulated genes in WS hMSCs restored upon Que or VC treatment. (C) Venn diagram and heatmap comparing upregulated genes in Que- or VC-treated WS hMSCs. (D) GO enrichment analysis for commonly upregulated genes in Que- or VC-treated WS hMSCs (top), genes only upregulated in Que-treated WS hMSCs (middle), and genes only upregulated in VC-treated WS hMSCs (bottom). The number of enriched genes in each GO term is shown in the circles.

enriched in cell cycle, nuclear division and chromosome segregation pathways, consistent with the cellular phenotypes observed. Previous studies on Que have mainly focused on its anti-carcinogenic (Ansgar, 2013; Darband Saber et al., 2018), anti-inflammatory (Griffiths et al., 2016; Li et al., 2016a; Liu et al., 2017; Salvatore, 2010), anti-viral
(Chiow et al., 2016; Li et al., 2016a), as well as anti-oxidant effects (Sohn et al., 2018). However, the mechanisms of Que against human stem cell aging remain to be elucidated. For the first time, we showed that Que alleviated hMSC aging at least partially via the reduction of ROS, and restoration of 
A

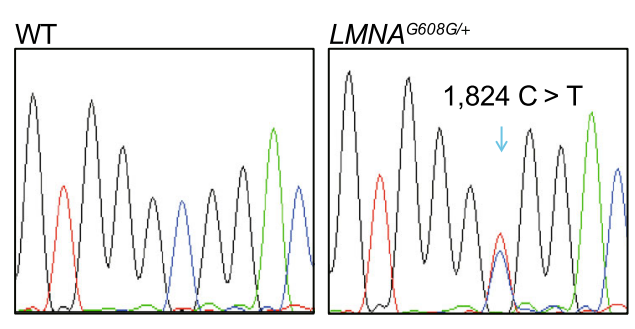

C

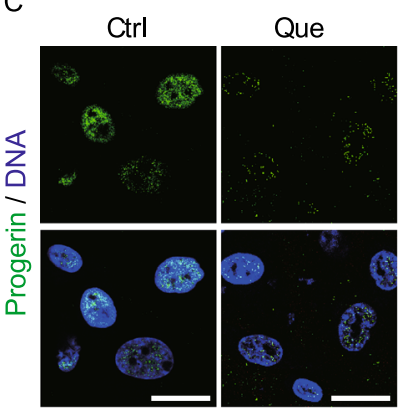

E
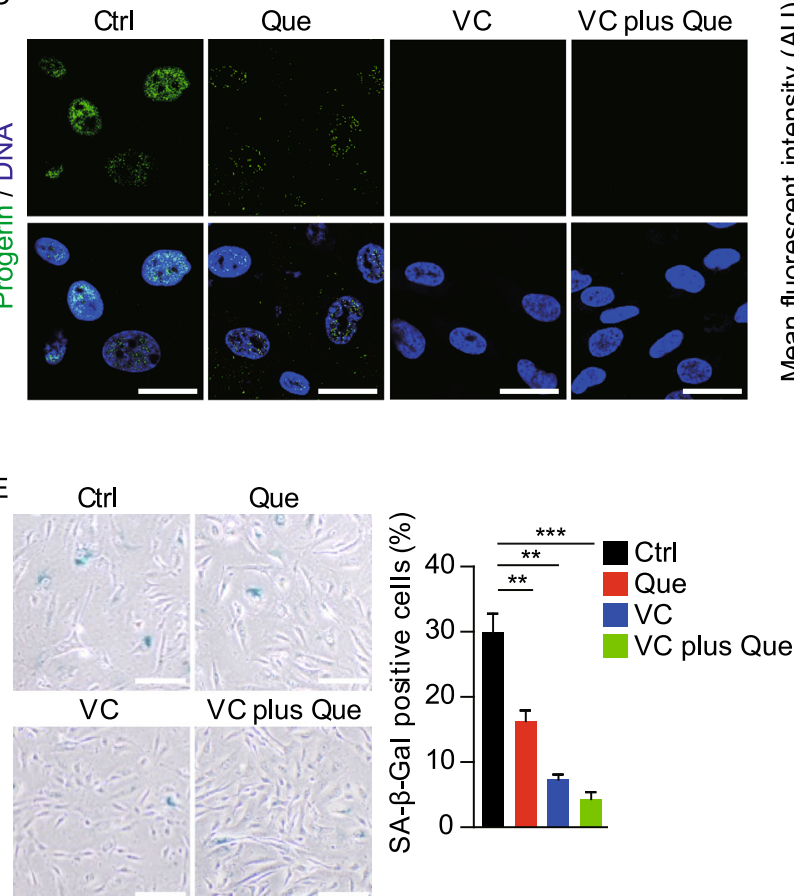

B

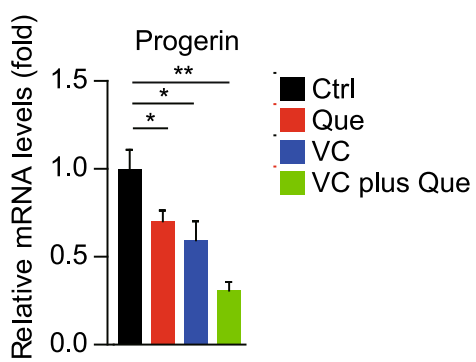

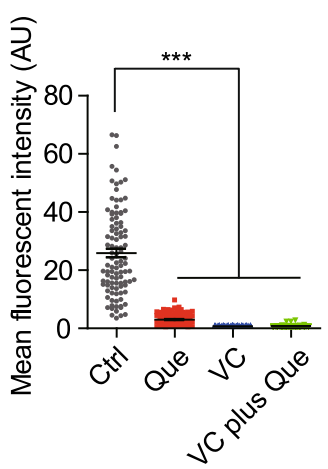

D
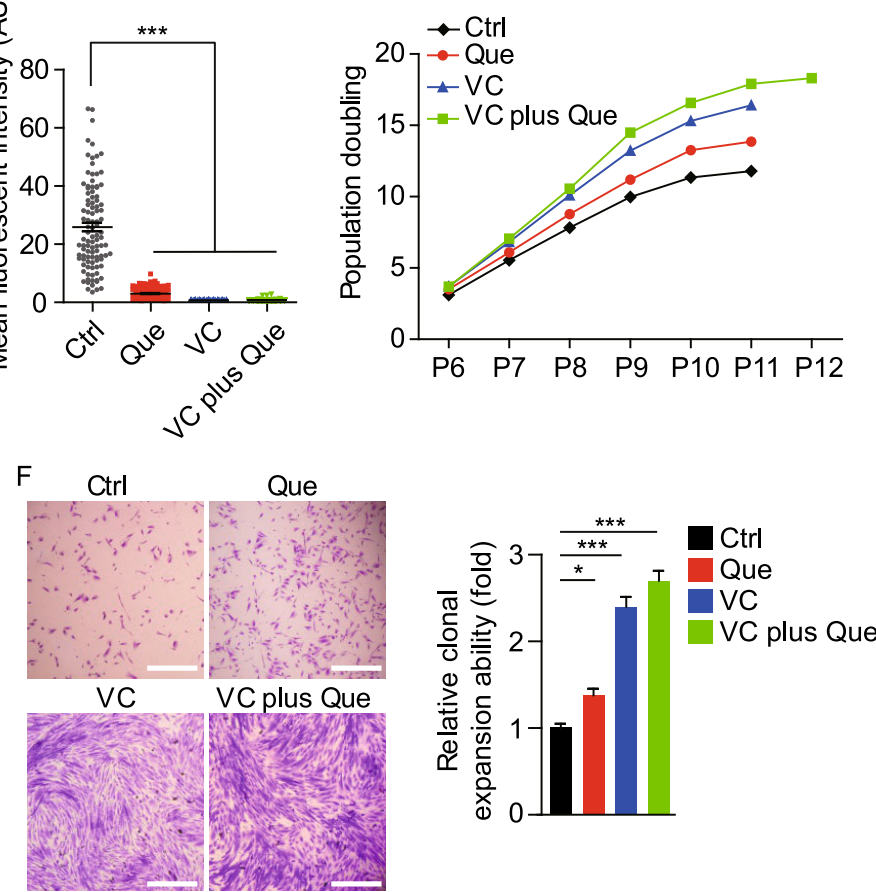

G

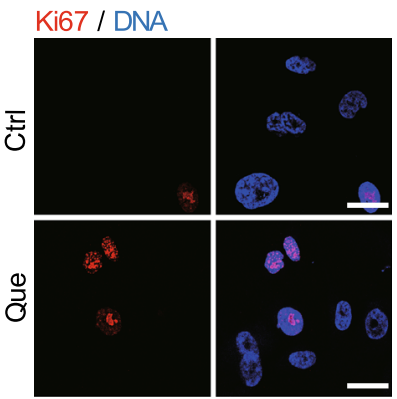

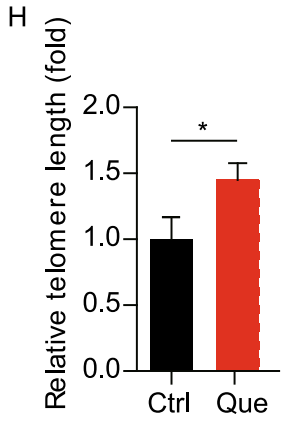
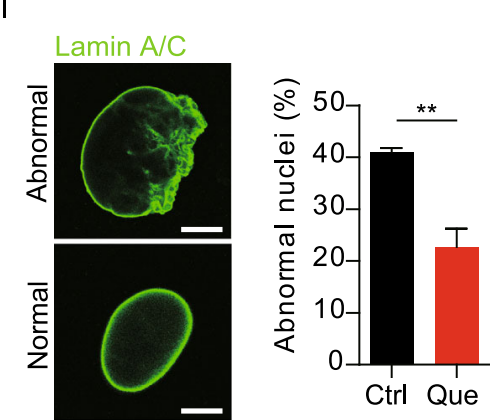
Figure 9. Que alleviated senescence in HGPS hMSCs with a synergistic effect with VC. (A) Confirmation of the heterozygous mutation of LMNA by DNA sequencing. (B) RTqPCR analysis of progerin mRNA expression in vehicle-, Que-, VC- and VC plus Que-treated HGPS hMSCs (passage 10). Data are shown as mean $\pm \operatorname{SEM}(n=3)$. ${ }^{*} P<0.05$ vs. Ctrl; ${ }^{* *} P<0.01$ vs. Ctrl. (C) Immunostaining of progerin in vehicle-, Que-, VC- and VC plus Que-treated HGPS hMSCs (passage 10), Scale bar, $25 \mu \mathrm{m}$. Mean fluorescence intensity on the right is shown as mean \pm SEM (cell number $\geq 300$ ). ${ }^{* * *} P<0.001$ vs. Ctrl. (D) Accumulative growth curve showing the population doubling of HGPS hMSCs upon the treatment of vehicle, Que, VC and VC plus Que $(n=3)$. (E) Analysis of SA- $\beta-G a l$ activity in vehicle-, Que-, VC- and VC plus Que-treated HGPS hMSCs. Left, representative images of SA- $\beta-$ Gal staining (passage 10); right, frequency of SA- $\beta$-Gal-positive cells. Data are shown as mean \pm SEM $(n=3) .{ }^{* *} P<0.01$ vs. Ctrl; ${ }^{* * *} P<0.001$ vs. Ctrl. Scale bar, $100 \mu \mathrm{m}$. (F) Clonal expansion ability of vehicle-, Que-, VC- and VC plus Que-treated HGPS hMSCs (passage 8) stained by crystal violet. Quantitative data are shown as mean $\pm \operatorname{SEM}(n=3) .{ }^{*} P<0.05$ vs. Ctrl; ${ }^{* *} P<0.001$ vs. Ctrl. Scale bar, $100 \mu \mathrm{m}$. (G) Immunostaining of Ki67 in vehicle- and Quetreated HGPS hMSCs (passage 10). Scale bar, $25 \mu \mathrm{m}$. Ki67positive cells are shown as mean \pm SEM (cell number $\geq 300$ ). ${ }^{*} P<0.05$ vs. Ctrl. (H) Quantitative PCR analysis of telomere length in vehicle- and Que-treated HGPS hMSCs (passage 10). Data are shown as mean $\pm \operatorname{SEM}(n=3)$. ${ }^{*} P<0.05$ vs. Ctrl. (I) Immunostaining of Lamin A/C in vehicle- and Que-treated HGPS hMSCs (passage 10). Quantifications of abnormal nuclei of HGPS hMSCs on the right are shown as mean \pm SEM $(n \geq 300) .{ }^{* *} P<0.01$ vs. Ctrl. Scale bar, $10 \mu \mathrm{m}$.

heterochromatin architecture, providing a new mechanism for Que-mediated geroprotection.

We have previously shown that VC alleviates aging defects in WS hMSCs by decreasing oxidative stress, preventing telomere attrition, suppressing excessive secretion of inflammatory factors, as well as reorganizing nuclear lamina and heterochromatin (Li et al., 2016b). Our comparative analysis of the RNA-seq data in WS hMSCs illustrated the mechanistic commonalities and differences of the geroprotective effects by Que and VC, with the commonly upregulated genes mainly enriched in gene terms related to cell cycle, chromatin condensation and anti-oxidation. Likewise, we observed that Que restored heterochromatin architecture and decreased ROS levels in WS hMSCs. It has been shown that increased ROS levels lead to heterochromatin disorganization. Therefore, in our study decreased ROS levels may also contribute to the restoration of heterochromatin architecture. In addition, we have recently demonstrated that, the premature aging phenotypes in HGPS hMSCs partially attributes to the repression of NRF2mediated anti-oxidative response, whereas the reactivation of NRF2 reverses the nuclear defects in HGPS cells and restores their in vivo viability in mice (Fang et al., 2018;
Kubben et al., 2016). Interestingly, Que is reported as an activator of NRF2-mediated anti-oxidative pathway (Bahar et al., 2017; Dai et al., 2018; Gao et al., 2018; Kim et al., 2018). Consistently, by RNA-seq analysis we identified that Que upregulated a series of anti-oxidative genes including GSTP1, GSR and GSTM1, all of which are NRF2 targets, indicating that Que may exert anti-oxidative effect via the activation of NRF2. Furthermore, despite of the multiple mechanistic commonalities of the geroprotective effects, Que-specific upregulated genes were mostly enriched in response to stimulus and cell homeostasis and the VCspecific ones in DNA replication, DNA repair and telomere maintenance, raising the possibility that Que and VC in combination might exhibit a synergistic geroprotective effect.

In this study, we showed that Que and VC exerted cooperative effects attenuating accelerated senescence in HGPS hMSCs. Besides VC, other geroprotective chemicals could also strengthen the biological activity of Que. For example, resveratrol and Que exhibit synergistic effects in the restoration of genes in pathways of glucose/lipid metabolism, liver function, cardiovascular system, and inflammation/immunity in mice fed with high-fat diet (Zhou et al., 2012). Moving forward, how to maximize the geroprotective effects of Que by combining with other components awaits further investigation.

In summary, we screened a natural product library in WS hMSCs and identified Que as a geroprotective agent against premature and physiological human aging through detailed mechanistic studies and multi-layer evaluations in WS, HGPS and physiological-aging hMSCs. We have also elucidated the transcriptional commonalities and differences in the geroprotective effects by Que and VC in WS hMSCs. In addition, given the multi-differentiation potentials of human pluripotent stem cells (hPSCs) and hMSCs, our platform with WS and HGPS hPSCs, hMSCs, and their derivatives provides a powerful tool for mechanistic studies and drug screening in various cell types, such as cardiovascular cells that are widely affected in WS and/or HGPS syndromes.

\section{MATERIALS AND METHODS}

Cell culture and differentiation

$W R N^{-1-} \mathrm{hMSC}$ and $L M N A^{\mathrm{G} 608 \mathrm{G} /+} \mathrm{hMSCs}$ were obtained via the directed differentiation of $W R N^{1-} \mathrm{hESCs}$ and $L M N A^{\mathrm{G} 608 \mathrm{G} /+} \mathrm{hESCs}$ that we previously established (Wu et al., 2018; Zhang et al., 2015). hESCs were maintained on mitomycin C-inactivated mouse embryonic fibroblast (MEF) feeder in hESC culture medium containing 80\% DMEM/F12 (Gibco), 20\% Knockout Serum Replacement (Gibco), $10 \mathrm{ng} / \mathrm{mL}$ FGF2 (Joint Protein Central), $0.1 \mathrm{mmol} / \mathrm{L}$ non-essential amino acids (NEAA, Gibco), $2 \mathrm{mmol} / \mathrm{L}$ GlutaMAX (Gibco) and $55 \mu \mathrm{mol} / \mathrm{L} \beta$-mercaptoethanol (Invitrogen). hESCs used in this study were also cultured on Matrigel (BD Biosciences) with mTeSR medium (STEMCELL Technologies). The human MSCs were cultured in hMSC culture medium containing 90\% a-MEM + Glutamax (Gibco), 10\% fetal bovine serum (FBS, Gemcell, Lot 
A

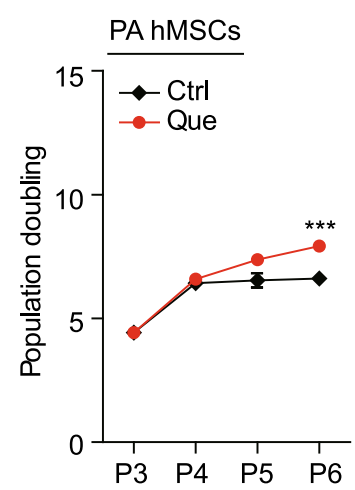

C

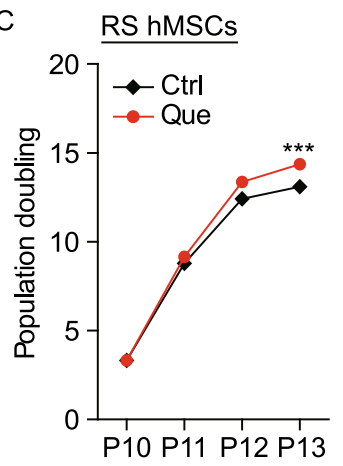

B

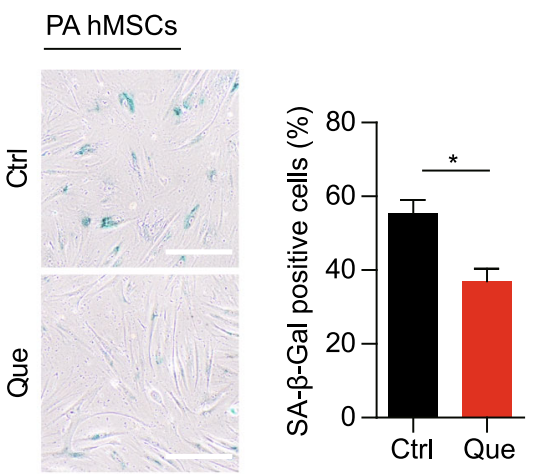

D RS hMSCs

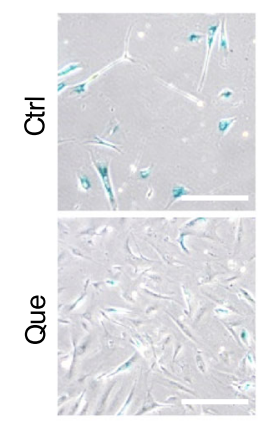

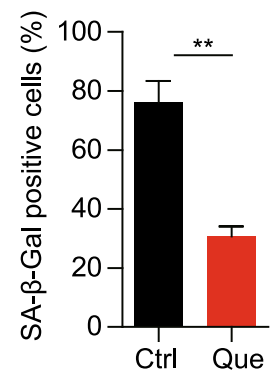

E $\quad \underline{\text { RS hMSCs }}$
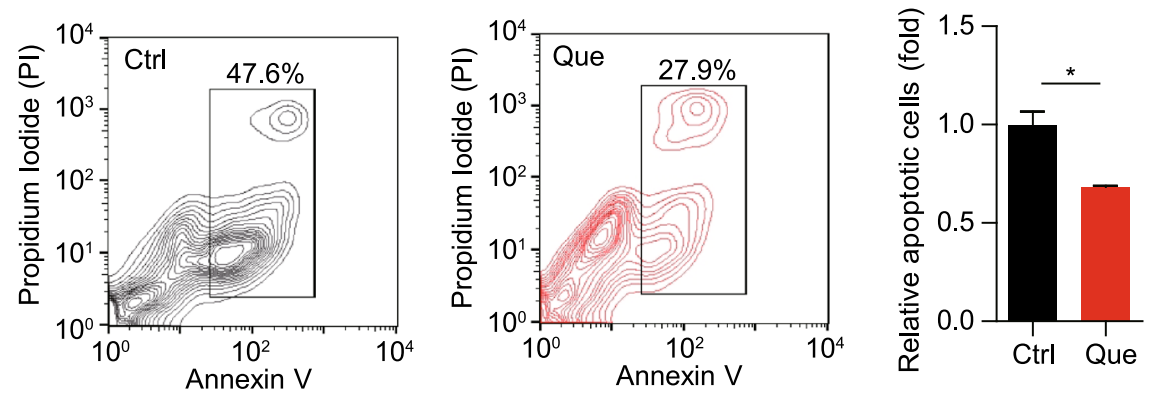

Figure 10. Que alleviated senescent phenotypes in physiological-aging (PA) hMSCs and replicative-senescent (RS) hMSCs. (A) Accumulative growth curve showing the population doubling of vehicle- and Que-treated PA hMSCs $(n=3)$. ${ }^{* \star *} P<0.001$ vs. Ctrl. (B) Analysis of SA- $\beta-$ Gal activity in vehicle- and Que-treated PA hMSCs (passage 5). Left, representative images of SA- $\beta-G a l$ staining; right, frequency of SA- $\beta$-Gal-positive cells. Data are shown as mean \pm SEM $(n=3)$. ${ }^{*} P<0.05$ vs. Ctrl. Scale bar, $100 \mu \mathrm{m}$. (C) Accumulative growth curve showing the population doubling of vehicle- and Que-treated RS hMSCs $(n=3)$. ${ }^{* *} P<0.001$ vs. Ctrl. (D) Analysis of SA- $\beta$-Gal activity in vehicle- and Que-treated RS hMSCs (passage 13). Left, representative images of SA- $\beta$-Gal

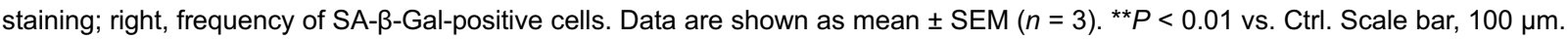

(E) Cell apoptosis assay in vehicle- and Que-treated RS hMSCs (passage 13). Quantitative data on the right are shown as mean \pm $\operatorname{SEM}(n=3) .{ }^{*} P<0.05$ vs. Ctrl.

A77E01F), $1 \mathrm{ng} / \mathrm{mL}$ FGF2 (Joint Protein Central) and 1\% penicillin/ streptomycin (Gibco).

Differentiation of hESCs into mesenchymal stem cells (MSCs) was performed as described previously (Duan et al., 2015; Fu et al., 2016; Wang et al., 2018a; Wang et al., 2018b; Zhang et al., 2015). Briefly, embryoid bodies were cultured in matrigel-coated plates with hMSC differentiation medium ( $\alpha$-MEM with GlutaMAX (Gibco), 10\%
FBS (Gemcell, Lot A77E01F), 10 ng/mL FGF2 (Joint Protein Central), $5 \mathrm{ng} / \mathrm{mL}$ TGF $\beta$ (Stemimmune LLC) and $1 \%$ penicillin/streptomycin (Gibco)). After about 10 days, the confluent MSC-like cells were passaged on gelatin-coated plate and cultured in hMSC culture medium containing $90 \%$ a-MEM with Glutamax (Gibco), 10\% fetal bovine serum (FBS, Gemcell, Lot A77E01F), 1 ng/mL FGF2 (Joint Protein Central) and 1\% penicillin/streptomycin (Gibco). 


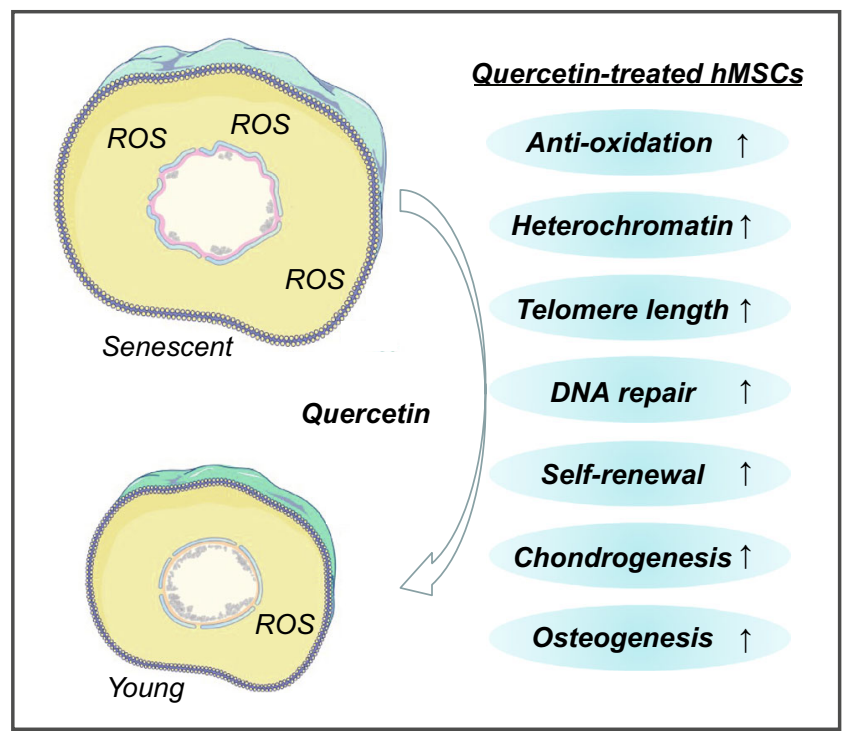

Figure 11. A proposed model illustrating the geroprotective effects of Que in hMSCs.

In vitro differentiation potentials of WS hMSCs into osteoblasts, chondrocytes and adipocytes (Pan et al., 2016; Wu et al., 2018; Zhang et al., 2015) were evaluated by Von Kossa (Abcam), toluidine blue (Sigma) and oil red O (Sigma) stainings, respectively.

\section{Preparation of physiological-aging hMSCs}

Physiological-aging (PA) hMSCs were isolated from gingiva of a male individual at 56 years old. In details, a piece of tissue (sized approximately by $7 \mathrm{~mm} \times 4 \mathrm{~mm} \times 2 \mathrm{~mm}$ ) was obtained from the gingival lamina propria of free gingiva after the removal of the gingival epithelium. The tissue was washed with PBS and minced into small pieces, followed by digestion with collagenase at $37^{\circ} \mathrm{C}$ for 30 $\mathrm{min}$. The hMSCs were collected upon centrifugation at 1,000 rpm for $5 \mathrm{~min}$ and were cultured in hMSC culture medium.

\section{Fluorescence-activated cell sorting (FACS)}

The human MSCs were purified by sorting against CD73, CD90 and CD105 triple positive cells using FACS. In details, hMSCs were digested using TrypLE Express (Gibco) and washed twice by PBS. Cells were incubated with primary antibody diluted with $10 \%$ FBS in PBS for $30 \mathrm{~min}$ at room temperature and sorted by using flow cytometer (BD FACSAria IIlu).

\section{Screening of a natural product library}

A total of 133 compounds of a natural product library (Selleck, L1400) were purchased and screened in WS hMSCs initially at 1 $\mu \mathrm{mol} / \mathrm{L}$. WS hMSCs (passage 5) were seeded into drug-stamped 96-well plates at a density of 3,000 cells per well. Culture medium with different kinds of compounds at $1 \mu \mathrm{mol} / \mathrm{L}$ was changed every other day. Seven days after the drug treatment, cell proliferation was measured by MTS assay (Promega). Values of quintic treatment wells were averaged and normalized to quintic control wells.
The top candidate compounds were further screened by a 30-day treatment at four different concentrations $(100,250,500$ and 1,000 $\mathrm{nmol} / \mathrm{L}$ ). WS hMSCs (passage 5) were seeded into 6-well plates at a density of 30,000 cells per well. Culture medium with different kinds of candidate compounds was changed every other day. The relative cell proliferative abilities of WS hMSCs (passage 7) treated with each compound at different concentrations were analyzed at day 30 .

\section{Determination of optimal Que concentration}

WS hMSCs (passage 5) were seeded into 96-well plates at a density of 3,000 cells per well and treated with different concentrations of quercetin hydrate (Que) (TCl, P0042). Culture medium with Que was changed every other day. Seven days after the drug treatment, cell proliferation was measured by MTS assay (Promega). Values of quintic treatment wells were averaged and normalized to quintic control wells and processed by calculating the $\log _{2}$ (Que/vehicle control).

\section{Senescence-associated $\beta$-galactosidase staining}

Senescence-associated $\beta$-galactosidase (SA- $\beta$-Gal) staining was performed as described previously (Debacq-Chainiaux et al., 2009). Briefly, hMSCs were washed in PBS and fixed at room temperature for $3 \mathrm{~min}$ in $2 \%$ formaldehyde and $0.2 \%$ glutaraldehyde. Fixed cells were then stained with freshly prepared staining solution for SA- $\beta$ Gal activity at $37{ }^{\circ} \mathrm{C}$ overnight. Images were taken and the percentage of positive cells was calculated.

\section{Clonal expansion assay}

Single-cell clonal expansion assay was carried out as described (Duan et al., 2015; Wu et al., 2018). Briefly, WS hMSCs were plated at a density of 2,000 cells per well in gelatin coated 12-well plate and treated with vehicle and Que. The cell density was calculated after crystal violet staining. 


\section{Western blotting}

$1 \times 10^{6}$ cells were collected and lysed with $100 \mu \mathrm{L}$ RIPA buffer $(0.1 \%$ SDS, $50 \mathrm{mmol} / \mathrm{L}$ Tris- $\mathrm{HCl}(\mathrm{pH} 7.5), 1 \% \mathrm{NP}-40,0.5 \%$ sodium deoxycholate, $150 \mathrm{mmol} / \mathrm{L} \mathrm{NaCl}$ ) supplemented with $\mathrm{NaVO}_{4}, \mathrm{NaF}$ and the protease-inhibitor mixture (Roche). BCA kit (Thermo Fisher Scientific) was used for measurement of protein concentration. 20 $\mu \mathrm{g}$ proteins for each sample were separated by SDS-PAGE and transferred onto a PVDF membrane (Millipore). The membrane was incubated with primary antibodies and then HRP-conjugated secondary antibodies. The quantification was performed with Image Lab software for ChemiDoc XRS system (Bio-Rad).

\section{Quantitative real-time PCR (RT-qPCR)}

Total RNAs were extracted using TRIzol Reagent (Invitrogen). 1-2 $\mu \mathrm{g}$ of total RNAs were converted to cDNA by using GoScript Reverse Transcription System (Promega). Quantitative real-time PCR was performed with iTaq Universal SYBR Green Super mix (BioRad) on a CFX384 Real-Time PCR system (Bio-Rad). Data were normalized to $18 \mathrm{~S}$ rRNA transcript and calculated using the $\Delta \triangle \mathrm{Cq}$ method. Quantitative RT-PCR primers used were listed in Table S4.

\section{Immunofluorescence microscopy}

Cells were prepared as previously reported (Wu et al., 2018). Briefly, hMSCs were seeded on microscope coverslips and fixed with $4 \%$ formaldehyde in PBS for $30 \mathrm{~min}$, permeabilized with $0.4 \%$ Triton X-100 in PBS for 30 min, and blocked with $10 \%$ donkey serum in PBS for $1 \mathrm{~h}$. The hMSCs on coverslips were incubated with primary antibodies (diluted with $1 \%$ donkey serum in PBS) at $4{ }^{\circ} \mathrm{C}$ overnight, and then incubated with fluorescence-labeled secondary antibodies (diluted with 1\% donkey serum in PBS) at room temperature for $1 \mathrm{~h}$. Hoechst 33342 (Invitrogen) was used to Stain nuclear DNA.

\section{In vivo stem cell retention analysis}

WS hMSCs implantation was performed as previously described (Zhang et al., 2015). In brief, $5 \times 10^{5}$ luciferase-expressing WS hMSCs were pretreated with vehicle or $100 \mathrm{nmol} / \mathrm{L}$ Que for 30 days and then implanted into the middle of the tibialis anterior muscle of immunodeficient mice. Five days after the implantation, mice were anaesthetized and injected with D-luciferin solution (Gold-Bio, luck-500). After $15 \mathrm{~min}$ the in vivo luciferase activity of each mouse was determined by the IVIS lumina system (PerkinElmer). Luminescence intensity was normalized to luciferase intensity of hMSCs right before the implantation. All the animal experiments performed in this study were approved by the Institute of Biophysics, Chinese Academy of Sciences.

\section{Fat pad vessel formation assay}

Fat pad transplantation was performed as previously described (Yu et al., 2016). Freshly collected vehicle- or Que-treated WS hMSCs (passage 7) were resuspended in Matrigel mixture consisting of 50\% Matrigel, 20\% FBS in PBS, and 0.01\% Trypan Blue (Sigma). $1.5 \times$ $10^{5}$ cells in $15 \mu \mathrm{L}$ volume was injected into the fat pads of 3 -weekold female non-obese diabetic (NOD) scid mice. Fat pads were harvested 4 weeks post-transplantation for measuring donor-derived vessel regeneration by immunofluorescent staining.

\section{RNA library construction and RNA sequencing}

WS hMSCs were treated with vehicle or Que from passage 5 and collected at passage 7 for RNA-seq analysis using Illumina sequencing platform. RNA sequencing libraries were prepared as previously reported ( $\mathrm{Li}$ et al., 2016b; Zhang et al., 2015). Briefly, RNA integrity was examined by the Bioanalyzer 2100 system (Agilent Technologies). Sequencing libraries were constructed using NEBNext UltraTM RNA Library Prep Kit for Illumina (NEB) and sequenced on Illumina Hiseq $X$ Ten platform.

\section{RNA-seq data processing}

The pipeline of RNA-seq data processing has been described previously (Zhang et al., 2015). In brief, sequencing reads were trimmed and mapped to hg19 human genome using hisat2 software (v2.0.4) (Kim et al., 2015). The transcriptional expression level of each gene was counted by HTSeq (v0.6.1) (Anders et al., 2015). Differentially expressed genes (DEGs) were computed using DESeq2 at a cutoff adjust $P$ value (Benjamini-Hochberg) less than 0.05 and $\mid \log _{1.5}$ (fold change)| more than 1 (Love et al., 2014). The correlation between replicates of each sample was evaluated by the Pearson correlation coefficient $(R)$, which was based on DESeq2 regularized-logarithm (rlog) normalized read count. Gene Ontology (GO) and pathway enrichment analysis was conducted by ToppGene (Chen et al., 2009). Protein-protein interaction networks of differentially expressed genes was drawn based on the STRING database (Szklarczyk et al., 2017).

Transcription levels of aging- and longevity-associated genes as well as the NRF2 target genes were analyzed as below. The agingand longevity-associated genes were identified according to the Human Ageing Genomic Resources (HAGR) database and NRF2 target genes from the C3 transcription factor targets in Molecular Signatures Database v6.0 (MSigDB, GSEA) with the addition of other NRF2 genes that have been previously reported (Kubben et al., 2016; Reszka et al., 2015; Tacutu et al., 2013). Genes with an adjust $P$ value (Benjamini-Hochberg) less than 0.05 were analyzed and $P$ values were calculated by Two-sample Kolmogorov-Smirnov test.

The RNA-seq data have been deposited to the NCBI Gene Expression Omnibus (GEO) database with accession number GSE116166.

\section{Antibodies}

Primary antibodies for FACS were anti-CD73-PE (550741, 1:100), anti-CD90-FITC (555595, 1:200) from Biosciences and anti-CD105APC (17-1057, 1:100) from eBioscience. Primary antibodies for Western blot were anti-WRN (sc-5629, 1:500), anti- $\beta$-Actin (sc130301, 1:3,000), anti- $\beta-T u b u l i n$ (sc-5274, 1:3,000) from Santa Cruz Biotechnology, anti-P21 (2947, 1:2,000), anti-HP1y $(2619,1: 1,000)$ from Cell Signaling Technology, anti-LAP2 $\beta(611000,1: 2,000)$ and anti-P16 (4828, 1:200) from BD Bioscience. Antibodies for immunofluorescent staining were anti-hSMA (ZM-0003) from ZSGBBio, anti-Progerin (sc-81611, 1:50), anti-Lamin A/C (sc-7293, 1:200) from Santa Cruz Biotechnology, anti-HP1y (2619, 1:500) from Cell Signaling Technology, anti-53BP1 (A300-273A, 1:500) from Bethyl Laboratories, anti-y-H2AX $(05-636,1: 500)$ from Millipore, anti- 
LAP2 $\beta$ (611000, 1:500), anti-hCD31 (555445, 1:200) from BD Bioscience, and anti-Ki67 (VP-RM04, 1:1,000) from Vector.

\section{Statistical analysis}

Student's $t$-test was used for statistical analysis. Data are presented as mean \pm SEM. $P<0.05$ is considered statistically significant.

\section{ACKNOWLEDGMENTS}

We thank Lei Bai, Ruijun Bai, Mingming Wei, Qun Chu, and Shikun Ma for administrative assistance, to Junying Jia (IBP, CAS) and Shuang Sun (IBP, CAS) for their useful help in FACS. We thank Shuo Guo (IBP, CAS), Xinyi Wu (IBP, CAS) for breeding and management of laboratory animals and Lei Zhou (IBP, CAS) for providing veterinary care. We are grateful to Can Peng (IBP, CAS) for TEM samples preparation and Tongxin Niu at HPC-Service Station in Center for Biological Imaging (IBP, CAS) for bioinformatic analysis. This work was supported by the National Key Research and Development Program of China (2017YFA0103304), the Strategic Priority Research Program of the Chinese Academy of Sciences (XDA16010100), the National Key Research and Development Program of China (2015CB964800, 2017YFA0102802, 2014CB910503 and 2018YFA0107203), the National High Technology Research and Development Program of China (2015AA020307), the National Natural Science Foundation of China (Grant Nos. 31671429, 91749202, 91749123, 81625009, 81330008, 81371342, 81471414, 81422017, 81601233, 81671377, $31601109,31601158,81771515$ and 81701388$)$, Program of Beijing Municipal Science and Technology Commission (Z151100003 915072), Key Research Program of the Chinese Academy of Sciences (KJZDEW-TZ-L05), Beijing Municipal Commission of Health and Family Planning (PXM2018_026283_000002), and Advanced Innovation Center for Human Brain Protection (117212).

\section{ABBREVIATIONS}

hESCs, human embryonic stem cells; HGPS, Hutchinson-Gilford progeria syndrome; hMSCs, human mesenchymal stem cells; Que, Quercetin; MTS, 3-(4,5-dimethylthiazol-2-yl)-5-(3-carboxymethoxyphenyl)-2-(4-sulfophenyl)-2H-tetrazolium, inner salt; $\mathrm{PA}$, physiological-aging; ROS, reactive oxygen species; RS, replicative-senescent; SA- $\beta$-Gal, senescence-associated- $\beta$ galactosidase; SASP, senescence-associated secretory phenotype; TEM, transmission electron microscope; VC, Vitamin C; WS, Werner syndrome.

\section{COMPLIANCE WITH ETHICS GUIDELINES}

The authors declare no conflict of interest.

OPEN ACCESS This article is distributed under the terms of the Creative Commons Attribution 4.0 International License (http:// creativecommons.org/licenses/by/4.0/), which permits unrestricted use, distribution, and reproduction in any medium, provided you give appropriate credit to the original author(s) and the source, provide a link to the Creative Commons license, and indicate if changes were made.

\section{REFERENCES}

Anders S, Pyl PT, Huber W (2015) HTSeq-a Python framework to work with high-throughput sequencing data. Bioinformatics 31:166-169

Ansgar B (2013) Inhibition of mTOR Signaling by Quercetin in Cancer Treatment and Prevention. Anticancer Agents Med Chem 13:1025-1031

Bahar E, Kim J-Y, Yoon H (2017) Quercetin Attenuates ManganeseInduced Neuroinflammation by Alleviating Oxidative Stress through Regulation of Apoptosis, iNOS/NF-KB and HO-1/Nrf2 Pathways. Int J Mol Sci 18:1989

Benayoun BA, Pollina EA, Brunet A (2015) Epigenetic regulation of ageing: linking environmental inputs to genomic stability. Nat Rev Mol Cell Biol 16:593-610

Burtner CR, Kennedy BK (2010) Progeria syndromes and ageing: what is the connection? Nature Reviews Molecular Cell Biology 11:567

Campisi J (2013) Aging, Cellular Senescence, and Cancer. Annual review of physiology 75:685-705

Chang J, Wang Y, Shao L, Laberge R-M, Demaria M, Campisi J, Janakiraman K, Sharpless NE, Ding S, Feng W et al (2016) Clearance of senescent cells by ABT263 rejuvenates aged hematopoietic stem cells in mice. Nature medicine 22:78-83

Chen J, Bardes EE, Aronow BJ, Jegga AG (2009) ToppGene Suite for gene list enrichment analysis and candidate gene prioritization. Nucl Acids Res 37:W305-W311

Chiow KH, Phoon MC, Putti T, Tan BKH, Chow VT (2016) Evaluation of antiviral activities of Houttuynia cordata Thunb. extract, quercetin, quercetrin and cinanserin on murine coronavirus and dengue virus infection. Asian Pacific Journal of Tropical Medicine 9:1-7

Chondrogianni N, Kapeta S, Chinou I, Vassilatou K, Papassideri I, Gonos ES (2010) Anti-ageing and rejuvenating effects of quercetin. Experimental Gerontology 45:763-771

Dai Y, Zhang H, Zhang J, Yan M (2018) Isoquercetin attenuates oxidative stress and neuronal apoptosis after ischemia/reperfusion injury via Nrf2-mediated inhibition of the NOX4/ROS/NFkappaB pathway. Chem Biol Interact 284:32-40

Darband Saber G, Kaviani M, Yousefi B, Sadighparvar S, Pakdel Firouz G, Attari Javad A, Mohebbi I, Naderi S, Majidinia M (2018) Quercetin: A functional dietary flavonoid with potential chemopreventive properties in colorectal cancer. Journal of Cellular Physiology 233:6544-6560

Debacq-Chainiaux F, Erusalimsky JD, Campisi J, Toussaint O (2009) Protocols to detect senescence-associated beta-galactosidase (SA-betagal) activity, a biomarker of senescent cells in culture and in vivo. Nat Protoc 4:1798-1806

Duan S, Yuan G, Liu X, Ren R, Li J, Zhang W, Wu J, Xu X, Fu L, Li Y (2015) PTEN deficiency reprogrammes human neural stem cells towards a glioblastoma stem cell-like phenotype. Nature Communications 6:10068

Fang J, Yang J, Wu X, Zhang G, Li T, Wang X, Zhang H, Wang C, Liu G-H, Wang L (2018) Metformin alleviates human cellular aging by upregulating the endoplasmic reticulum glutathione peroxidase 7. Aging Cell 17(4):e12765

Fu L, Xu X, Ren R, Wu J, Zhang W, Yang J, Ren X, Wang S, Zhao Y, Sun $L$ et al (2016) Modeling xeroderma pigmentosum associated 
neurological pathologies with patients-derived iPSCs. Protein \& Cell 7:210-221

Gao W, Pu L, Chen M, Wei J, Xin Z, Wang Y, Yao Z, Shi T, Guo C (2018) Glutathione homeostasis is significantly altered by quercetin via the Keap1/Nrf2 and MAPK signaling pathways in rats. Journal of Clinical Biochemistry and Nutrition 62:56-62

Griffiths K, Aggarwal BB, Singh RB, Buttar HS, Wilson D, De Meester F (2016) Food Antioxidants and Their Anti-Inflammatory Properties: A Potential Role in Cardiovascular Diseases and Cancer Prevention. Diseases 4:28

Harhouri K, Frankel D, Bartoli C, Roll P, De Sandre-Giovannoli A, Lévy N (2018) An overview of treatment strategies for Hutchinson-Gilford Progeria Syndrome. Nucleus 9(1):246-257

Harley CB (1991) Telomere loss: mitotic clock or genetic time bomb? Mutation Research 256:271-282

Hennekam Raoul CM (2006) Hutchinson-Gilford progeria syndrome: Review of the phenotype. American Journal of Medical Genetics Part A 140A:2603-2624

Kampkötter A, Timpel C, Zurawski RF, Ruhl S, Chovolou Y, Proksch $P$, Wätjen W (2008) Increase of stress resistance and lifespan of Caenorhabditis elegans by quercetin. Comparative Biochemistry and Physiology Part B: Biochemistry and Molecular Biology 149:314-323

Kim D, Langmead B, Salzberg SL (2015) HISAT: a fast spliced aligner with low memory requirements. Nature methods 12:357360

Kim Y, Kim CS, Joe Y, Chung HT, Ha TY, Yu R (2018) Quercetin Reduces Tumor Necrosis Factor Alpha-Induced Muscle Atrophy by Upregulation of Heme Oxygenase-1. Journal of medicinal food 21:551-559

Kreienkamp R, Croke M, Neumann MA, Bedia-Diaz G, Graziano S, Dusso A, Dorsett D, Carlberg C, Gonzalo S (2016) Vitamin D receptor signaling improves Hutchinson-Gilford progeria syndrome cellular phenotypes. Oncotarget 7:30018-30031

Kubben N, Zhang W, Wang L, Voss TC, Yang J, Qu J, Liu GH, Misteli T (2016) Repression of the antioxidant NRF2 pathway in premature aging. Cell 165(6):1361-1374

Kudlow BA, Kennedy BK, Monnat RJ Jr (2007) Werner and Hutchinson-Gilford progeria syndromes: mechanistic basis of human progeroid diseases. Nature Reviews Molecular Cell Biology 8:394

López-Otín C, Blasco MA, Partridge L, Serrano M, Kroemer G (2013) The Hallmarks of Aging. Cell 153:1194-1217

Labbé A, Turaga RV, Paquet ER, Garand C, Lebel M (2010) Expression profiling of mouse embryonic fibroblasts with a deletion in the helicase domain of the Werner Syndrome gene homologue treated with hydrogen peroxide. Bmc Genomics 11:127

Lebel M (2001) Werner syndrome: genetic and molecular basis of a premature aging disorder. Cellular and molecular life sciences : CMLS 58:857-867

Li Y, Yao J, Han C, Yang J, Chaudhry MT, Wang S, Liu H, Yin Y (2016a) Quercetin. Inflammation and Immunity. Nutrients 8:167

Li Y, Zhang W, Chang L, Han Y, Sun L, Gong X, Tang H, Liu Z, Deng $\mathrm{H}, \mathrm{Ye} \mathrm{Y}$ et al (2016b) Vitamin C alleviates aging defects in a stem cell model for Werner syndrome. Protein \& Cell 7:478-488
Liu GH, Barkho BZ, Ruiz S, Diep D, Qu J, Yang S-L, Panopoulos $A D$, Suzuki K, Kurian L, Walsh C et al (2011a) Recapitulation of premature aging with iPSCs from Hutchinson-Gilford progeria syndrome. Nature 472:221-225

Liu GH, Suzuki K, Qu J, Sancho-Martinez I, Yi F, Li M, Kumar S, Nivet E, Kim J, Soligalla RD et al (2011b) Targeted Gene Correction of Laminopathy-Associated LMNA Mutations in Patient-Specific iPSCs. Cell stem cell 8:688-694

Liu W, Zhang M, Feng J, Fan A, Zhou Y, Xu Y (2017) The Influence of Quercetin on Maternal Immunity, Oxidative Stress, and Inflammation in Mice with Exposure of Fine Particulate Matter during Gestation. International Journal of Environmental Research and Public Health 14:592

Lombard DB, Chua KF, Mostoslavsky R, Franco S, Gostissa M, Alt FW (2005) DNA Repair, Genome Stability, and Aging. Cell 120:497-512

Love MI, Huber W, Anders S (2014) Moderated estimation of fold change and dispersion for RNA-seq data with DESeq2. Genome biology 15:550

Murfuni I, Santis AD, Federico M, Bignami M, Pichierri P, Franchitto A (2012) Perturbed replication induced genome wide or at common fragile sites is differently managed in the absence of WRN. Carcinogenesis 33:1655

Ogrodnik M, Miwa S, Tchkonia T, Tiniakos D, Wilson CL, Lahat A, Day CP, Burt A, Palmer A, Anstee QM et al (2017) Cellular senescence drives age-dependent hepatic steatosis. Nature Communications 8:15691

Opresko PL, Cheng WH, von Kobbe C, Harrigan JA, Bohr VA (2003) Werner syndrome and the function of the Werner protein; what they can teach us about the molecular aging process. Carcinogenesis 24:791-802

Ozgenc A, Loeb LA (2006) Werner Syndrome, aging and cancer. Genome dynamics 1:206-217

Pan H, Guan D, Liu X, Li J, Wang L, Wu J, Zhou J, Zhang W, Ren R, Zhang W (2016) SIRT6 safeguards human mesenchymal stem cells from oxidative stress by coactivating NRF2. Cell Research 26:190-205

Polosak J, Kurylowicz A, Roszkowska-Gancarz M, Owczarz M, Puzianowska-Kuznicka M (2011) Aging is accompanied by a progressive decrease of expression of the WRN gene in human blood mononuclear cells. Journals of Gerontology 66:19

Ren R, Deng L, Xue Y, Suzuki K, Zhang W, Yu Y, Wu J, Sun L, Gong $X$, Luan $\mathrm{H}$ (2017a) Visualization of aging-associated chromatin alterations with an engineered TALE system. Cell Research 27:483-504

Ren R, Ocampo A, Liu GH, Izpisua Belmonte JC (2017b) Regulation of Stem Cell Aging by Metabolism and Epigenetics. Cell metabolism 26:460-474

Ren X, Lim S, Ji Z, Yuh J, Peng V, Smith MT, Zhang L (2011) Comparison of Proliferation and Genomic Instability Responses to WRN Silencing in Hematopoietic HL60 and TK6 Cells. Plos One 6:e14546

Reszka E, Wieczorek E, Jablonska E, Janasik B, Fendler W, Wasowicz W (2015) Association between plasma selenium level and NRF2 target genes expression in humans. Journal of Trace Elements in Medicine and Biology 30:102-106 
Salvatore C (2010) The Role of Quercetin, Flavonols and Flavones in Modulating Inflammatory Cell Function. Inflammation \& Allergy - Drug Targets (Discontinued) 9:263-285

Seki M, Otsuki M, Ishii Y, Tada S, Enomoto T (2008) RecQ family helicases in genome stability: lessons from gene disruption studies in DT40 cells. Cell Cycle 7:2472-2478

Shamanna RA, Croteau DL, Lee JH, Bohr VA (2017) Recent advances in understanding werner syndrome. F1000research 6:1779

Smith JR, Pereirasmith OM (1996) Replicative Senescence: Implications for in Vivo Aging and Tumor Suppression. Science 273:63-67

Sohn E-J, Kim JM, Kang S-H, Kwon J, An HJ, Sung J-S, Cho KA, Jang I-S, Choi J-S (2018) Restoring effects of natural anti-oxidant quercetin on cellular senescent human dermal fibroblasts. Am J Chin Med 46(4):1-21

Szklarczyk D, Morris JH, Cook H, Kuhn M, Wyder S, Simonovic M, Santos A, Doncheva NT, Roth A, Bork P et al (2017) The STRING database in 2017: quality-controlled protein-protein association networks, made broadly accessible. Nucl Acids Res 45:D362-D368

Tacutu R, Craig T, Budovsky A, Wuttke D, Lehmann G, Taranukha D, Costa J, Fraifeld VE, de Magalhaes JP (2013) Human Ageing Genomic Resources: integrated databases and tools for the biology and genetics of ageing. Nucleic Acids Res 41:D10271033

Uccelli A, Moretta L, Pistoia V (2008) Mesenchymal stem cells in health and disease. Nature reviews Immunology 8:726-736

Ullrich N, Gordon L (2015) Chapter 18 - Hutchinson-Gilford progeria syndrome. In: Islam MP, Roach ES (eds) Neurocutaneous Syndromes. Handbook of Clinical Neurology, vol 132. Elsevier, pp 249-264

Villeponteau B (1997) The heterochromatin loss model of aging. Experimental Gerontology 32:383-394

Wang P, Liu Z, Zhang X, Li J, Sun L, Ju Z, Li J, Chan P, Liu G-H, Zhang $W$ et al (2018a) CRISPR/Cas9-mediated gene knockout reveals a guardian role of NF-KB/RelA in maintaining the homeostasis of human vascular cells. Protein Cell. https://doi. org/10.1007/s13238-018-0560-5
Wang S, Hu B, Ding Z, Dang Y, Wu J, Li D, Liu X, Xiao B, Zhang W, Ren $R$ et al (2018b) ATF6 safeguards organelle homeostasis and cellular aging in human mesenchymal stem cells. Cell Discovery $4: 2$

Wu Z, Zhang W, Song M, Wang W, Wei G, Li W, Lei J, Huang Y, Sang $Y$, Chan $P$ et al (2018) Differential stem cell aging kinetics in Hutchinson-Gilford progeria syndrome and Werner syndrome. Protein \& Cell 9:333-350

Xu Z, Feng W, Shen Q, Yu N, Yu K, Wang S, Chen Z, Shioda S, Guo $Y$ (2017) Rhizoma Coptidis and Berberine as a Natural Drug to Combat Aging and Aging-Related Diseases via Anti-Oxidation and AMPK Activation. Aging and Disease 8:760-777

Yang J, Cai N, Yi F, Liu GH, Qu J, Izpisua Belmonte JC (2014) Gating pluripotency via nuclear pores. Trends in molecular medicine 20:1-7

Yang J, Li J, Suzuki K, Liu X, Wu J, Zhang W, Ren R, Zhang W, Chan P, Izpisua Belmonte JC et al (2017) Genetic enhancement in cultured human adult stem cells conferred by a single nucleotide recoding. Cell Res 27:1178-1181

Yu CE, Oshima J, Fu YH, Wijsman EM, Hisama F, Alisch R, Matthews S, Nakura J, Miki T, Ouais S (1996) Positional cloning of the Werner's syndrome gene. Science 272:258-262

Yu QC, Song W, Wang D, Zeng YA (2016) Identification of blood vascular endothelial stem cells by the expression of protein $C$ receptor. Cell Research 26:1079-1098

Zhang W, Li J, Suzuki K, Qu J, Wang P, Zhou J, Liu X, Ren R, Xu X, Ocampo A et al (2015) A Werner syndrome stem cell model unveils heterochromatin alterations as a driver of human aging. Science (New York, NY) 348:1160-1163

Zhou M, Wang S, Zhao A, Wang K, Fan Z, Yang H, Liao W, Bao S, Zhao L, Zhang $Y$ et al (2012) Transcriptomic and Metabonomic Profiling Reveal Synergistic Effects of Quercetin and Resveratrol Supplementation in High Fat Diet Fed Mice. Journal of Proteome Research 11:4961-4971

Zhu Y, Tchkonia T, Pirtskhalava T, Gower AC, Ding H, Giorgadze N, Palmer AK, Ikeno Y, Hubbard GB, Lenburg M et al (2015) The Achilles' heel of senescent cells: from transcriptome to senolytic drugs. Aging Cell 14:644-658 